\title{
Large-eddy simulation of particle-laden turbulent flows
}

\author{
M. BINI AND W. P. JONES \\ Mechanical Engineering Department, Imperial College London, Exhibition Road, \\ London SW7 2AZ, UK
}

(Received 8 November 2007 and in revised form 7 July 2008)

A large-eddy-based methodology for the simulation of turbulent sprays is discussed. The transport equations for the spatially filtered gas phase variables, in which source terms accounting for the droplet effects are added, are solved together with a probabilistic description of the liquid phase. The probabilistic approach for the liquid phase is based on the transport equation for the spatially filtered joint probability density function of the variables required in order to describe the state of the liquid phase. In this equation, unclosed terms representing the filtered Lagrangian rates of change of the variables describing the spray are present. General modelling ideas for subgrid-scale (SGS) effects are proposed. The capabilities of the approach and the validity of the closure models, with particular with respect to the SGS dispersion, are investigated through application to a dilute particle-laden turbulent mixing layer. It is demonstrated that the formulation is able to reproduce very closely the measured properties of both the continuous and dispersed phases. The large-eddy simulation (LES) results are also found to be entirely consistent with the experimentally observed characteristics of droplet-gas turbulence interactions. Consistent with direct numerical simulation (DNS) studies of isotropic turbulence laden with particles where the entire turbulence spectrum is found to be modulated by the presence of particles, the present investigation, which comprises the effects of particle transport upon the large-scale vortical structures of a turbulent shear flow, highlights what appears to be a selective behaviour; few large-scale frequencies gain energy whereas the remaining modes are damped.

\section{Introduction}

The ability to model and numerically simulate droplet-laden turbulent flows would lead to significant improvements in the design of a variety of engineering devices such as combustion chambers, engines, furnaces and many other industrial processes employing sprays. In a two-phase flow, a set of conservation equations can be written for each of the two phases. If a deterministic treatment is attempted then one major consequence is that the interface between the two phases would have to be determined as a part of the solution. This approach is certainly not practicable for a spray where a very large number of droplets is usually present. A more feasible strategy is to assume that the motion of a single droplet does not create significant additional turbulent fluctuations in the carrier gas (continuous phase) flow field. Such an assumption is justified in the majority of sprays where droplet sizes are typically in the range $1-100 \mu \mathrm{m}$ any new fluctuations induced in the gas phase by the droplet motion, i.e. the particle wakes, would then have length scales lying well within the 
viscous-dissipation-dominated scales of the continuous-phase turbulence spectrum. Thus any fluctuations generated by droplet motion would be quickly dissipated.

The reasoning above suggests that droplets can be treated as if they are effectively point sources and forces. This then implies the need for models which are able to mimic the mutual exchanges and interactions between the two phases. Various models are available in the literature for the determination of the mass and energy exchanges between a single droplet and the surrounding gas (see i.e. Lefebvre 1989; Sirignano 1999). These models represent, with varying degrees of complexity, the phase interactions involving mass and energy transfer driven by the dominant phenomenon of convection. The latter can be modelled accurately only when the relative dynamics of the moving droplets - the dispersed phase - and the gas or continuous phase are known. These dynamics are directly responsible for the particulate phase accumulation, dispersion and transport. For this reason the modelling of the momentum exchanges between the two phases can be identified as a key factor to be captured if reliable spray simulations are to be achieved.

In sprays, the momentum exchange between the two phases includes the effects of the turbulence upon the droplets motion as well as any turbulence modulation arising from that motion. Experimental findings (e.g. see Kobayashi et al. 1988; Lazaro \& Lasheras 1989; 1992a, b; Longmire \& Eaton 1992; Wen et al. 1992; Fessler, Kulick \& Eaton 1994) have confirmed the belief that the spectral characteristics of a particle-laden flow may differ substantially from that of a single-phase turbulent flow. Various studies have been dedicated to the investigation of the zones of preferential particle concentration: Lazaro \& Lasheras (1992a) and Longmire \& Eaton (1992) have shown that dense particles collect in the saddle regions between successive vortex rings; Maxey (1987), Squires \& Eaton $(1990,1991)$ and Wang \& Maxey $(1993)$ found that selective particle concentration is strongly dependent on the ratio of the particle inertial time to a turbulent characteristic time. Numerical studies concerning accumulation and dispersion of heavy particles in forced plane mixing layers have been carried out in a series of works: Martin \& Meiburg (1994); Raju \& Meiburg (1995); Marcu \& Meiburg (1996a,b); Marcu, Meiburg \& Raju (1996); they all reported the formation of highly concentrated particle streaks in the region between two vortices. The behaviour of heavy particles in homogeneous isotropic turbulence has been studied experimentally by Aliseda et al. (2002) and numerically by Bosse, Kleiser \& Meiburg (2006) where it is shown that the settling velocity is enhanced by the turbulence and that large temporal and spatial inhomogeneities in the particle concentration fields arise; the particles concentrate preferentially in certain regions of the flow.

Several works (e.g. Crowe 1982; Lazaro \& Lasheras 1989, 1992a) have demonstrated that the effect of the particle motions on the gas phase turbulence is significant only in the case of high particle to gas volume ratios and high mass loadings, typically at particle to gas volume ratios of the order of or greater than between $10^{-3}$ and $10^{-4}$. For flows characterized by small particle mass loading, the momentum loss or gain to turbulence is negligible. However, because of the preferential concentration of particles at the 'edge' of eddies, it is necessary to be aware that globally low mass loadings can lead to highly discontinuous and inhomogeneous fields of particle concentration (Squires \& Eaton 1990).

The effect of particles on turbulent flow structures is poorly understood. A common view is that the presence of particles provides extra dissipation, thus damping vortical structures. Gore \& Crowe (1989) suggested that turbulence will be augmented or suppressed depending on the ratio of particle dimension to a turbulent length scale. 
Elghobashi \& Truesdell (1993) concluded that 'the particles, due to their inertia impart their energy to the turbulent motion at high wavenumbers with a corresponding increase in the dissipation. The enhanced dissipation signals the smaller wavenumbers (larger scales), to supply more energy to the highly active small scales, at a rate higher than that of the particle-free case'. The overall energy spectrum is thus reduced at small wavenumbers and increased at high wavenumbers. These observations lead to the conclusion that, in the absence of energy sources such as shear in the mean flow, the energy of turbulent fluctuations will decay at a faster rate than in the particle-free flow. Boivin, Simonin \& Squires (1998) point out that DNS of isotropic decaying turbulence is not the appropriate framework for studying two-way coupling effects: the restriction to low Reynolds numbers and the limited range of scales limits a determination of whether two-way coupling is best described in terms of large- or small-scale variables. Ahmed \& Elghobashi (2000) showed how particles, by increasing the local velocity gradients, act to augment the local strain rate, which increases the turbulence energy dissipation rate. A preferential accumulation of particles in low-vorticity regions further increases the local velocity gradients, thus modifying the vorticity dynamics. Druzhinin (2001) analysed the modulation of isotropic turbulence by solid particles and found that micro-particles, for which the particle response time, $\tau_{p}$ was much less than the Kolmogorov time scale, $t_{K}$, resulted in an increase in the turbulence kinetic energy. In the case of particles with higher inertia, on the other hand, the net effect is a reduction of the turbulence kinetic energy. Ferrante \& Elghobashi (2003) have shown how micro-particles reduce the decay rate of turbulence kinetic energy whereas large particles enhance it compared to particle-free turbulence. In the transition zone, $\tau_{p} / t_{K} \approx 0.25$, Ferrante \& Elghobashi introduced the term ghost particles. Ghost particles kept the decay rate of the turbulent kinetic energy nearly identical to that of particle-free turbulence by modifying the energy spectrum in a unique way; the energy gained at high wavenumbers exactly balances the energy lost at small wavenumbers. The explanation offered was: 'micro-particles remain in their initial surrounding vortices with their trajectories almost aligned with those of the initial fluid points, but with higher inertia. Thus, they allow the vortical structures to retain their initial vorticity and strain rate for longer. As a result, both turbulence kinetic energy and viscous dissipation rate are larger than that of the particle-free turbulence at all times. Large particles cross the trajectories of fluid elements, enter new eddies, bringing an overall disorganization and thus enhancing the decay rate in comparison to the particle-free flow. Accordingly, the turbulence length- and time scales grow faster, and the turbulence kinetic energy and the strain rate decay faster than those in particle-free turbulence'.

The numerical modelling of a droplet-laden flow should ideally treat both the above-mentioned effects, modification of the turbulence characteristics due to the particles motion and particle dispersion due to the entire spectrum of turbulent fluctuations. In the context of large-eddy simulation (LES) two problems arise: (a) the droplet motions interact with and modify the smallest scales of the turbulence; and $(b)$ the turbulent fluctuations are not completely known and as a consequence a model is required in order to reproduce the effects of the unresolved turbulent scales on the particle dynamics. From the LES literature it is well known that in single-phase flows the main role of the model for the subgrid scale stress is to mimic the effect of the unresolved scales upon the resolved ones and thereby provide the correct amount of dissipation. The question of how the subgrid scale (SGS) models developed for single-phase flows may be adapted to droplet-laden flows has been addressed in the direct numerical simulation (DNS) studies of Bellan (2000), Okong'o \& Bellan (2000) 
and Miller \& Bellan (2000). An aspect of primary importance in dense spray flows is the influence of the droplet dynamics on the dissipation rate. Apart for the a priori analysis of Okong'o \& Bellan (2004), it appears that there has been only one LES (Yuu, Ueno \& Umekage 2001) in which the modelled SGS stress tensor has been modified to account for dispersed phase effects on the subgrid scale turbulence.

On the other hand, the modelling of the transport and dispersion of the particulate phase consists of formulating a suitable expression for the force felt by a travelling droplet as a function of the continuous phase velocity at the droplet position and the particle velocity. It must be noted here that no exact relation for such forces exists. The best known expression is that given by Maxey \& Riley (1983) for an isolated particle in a Stokes flow. This is the expression invariably adopted in deterministic DNS studies in which the entire gas-phase turbulent fluctuations are resolved and various interpolation schemes are used for the calculation of the gas velocity at the particle positions (Ferrante \& Elghobashi 2003; Okong'o \& Bellan 2004). In the context of LES, the gas-phase velocity is not completely known and if the force were to be evaluated solely in terms of the resolved velocity field, the effect of the unresolved fluctuations upon the particle motion would be omitted. In an LES of a dilute spray, the magnitude of any error associated with the use of an unmodified single phase SGS model is likely to be much smaller than that due to an inadequate representation of the dispersion effects of the unresolved gas fluctuations on the liquid dispersed phase, as is shown by Okong'o \& Bellan (2004). However, quoting Mashayek \& Pandya (2003), 'the above conclusion is despite the fact that the majority of the LES studies tend to (conveniently) neglect the subgrid velocity fluctuations'.

In fully developed turbulence, the effects of small scales upon particle acceleration should be treated with particular care. Measurements, by mean of optical devices, of the acceleration experienced by particles in a turbulent flow (e.g. Mordant et al. 2001; La Porta et al. 2002; Voth et al. 2002), provide evidence that particles are likely to experience accelerations which are many times higher than the acceleration standard deviation with a probability significantly larger than that corresponding to a Gaussian distribution. The problem of modelling sub-filter particle accelerations has been addressed by Bini \& Jones (2007) where a stochastic model, capable of reproducing the observed far from Gaussian behaviour, has been constructed.

\subsection{Scope and structure of the present work}

The main aim of the present work is to assess the capabilities of large-eddy simulation in representing a droplet-laden shear flow which is spatially developing. The approach followed involves a two-way coupling in which a probabilistic approach, adopted for the dispersed phase, explicitly accounts for subgrid-scale dispersion effects on the droplets. In Bini \& Jones (2007), a model for the LES unresolved fluctuations upon particle dynamics is formulated and it is shown how the model is capable of reproducing the experimentally observed heavy-tailed probability distribution of particle acceleration. In the present work, this model is applied in LES and the applicability of various alternative formulations, such as those of Miller \& Bellan (2000) and Okong'o \& Bellan (2004), are also reviewed and discussed. The structure of the paper is as follow: first, it will be shown how the separate probabilistic treatment of the dispersed phase, when coupled with the LES of the gas phase, can be viewed in the frame of a spatially filtered probability density function (PDF) transport equation. Secondly, the closure models required in the filtered PDF equation will be discussed with particular attention paid to the dispersion effects of the unresolved SGS scales. Thirdly, we will show how such an approach can reproduce the main features of the 
spatially developing mixing layer studied experimentally by Lazaro \& Lasheras (1989, $1992 a, b)$. In doing so, we will try to highlight the influence of LES inflow conditions on downstream development, showing how they can be treated and what their relative effects are, recalling that such dependencies have often constituted the reasons for switching the attention to the study of temporally evolving model flows. The LES results for the mixing layer are found to reproduce practically all the measured properties, and identical conclusions on particle transport, with respect to size and distribution, can be drawn from the LES and the experiments. Given this consistency, a further detailed analysis of the LES results is conducted using conventional and non-stationary spectral analysis with the aim of elucidating the physics underlying particle and turbulence interactions.

\section{Formulation}

\subsection{Spatially filtered balances for the gas phase}

As discussed in $\S 1$, the assumption of a particulate phase whose characteristic dimensions are smaller than the smallest turbulent length scales enables particles to be viewed, in respect of their influence on the gas phase, as a point source. This means that source terms will appear in the differential form of the conservation equations for the gas phase; these will represent the effects played by all the particles contained within the incremental fluid element for which the balances are written:

$$
\begin{gathered}
\frac{\partial \rho}{\partial t}+\frac{\partial\left(\rho u_{i}\right)}{\partial x_{i}}=S_{\text {mass }} \\
\frac{\partial\left(\rho u_{i}\right)}{\partial t}+\frac{\partial\left(\rho u_{i} u_{j}\right)}{\partial x_{j}}=-\frac{\partial p}{\partial x_{i}}+\frac{\partial \sigma_{i j}}{\partial x_{j}}+\rho f_{i}^{\text {ext }}+S_{\text {mom }, i} \\
\frac{\partial(\rho Y)}{\partial t}+\frac{\partial\left(\rho Y u_{j}\right)}{\partial x_{j}}=-\frac{\partial J_{j}}{\partial x_{j}}+S_{\text {species }}, \\
\frac{\partial(\rho e)}{\partial t}+\frac{\partial\left(\rho e u_{j}\right)}{\partial x_{j}}=\frac{\partial\left(p u_{j}\right)}{\partial x_{j}}-\frac{\partial q_{j}}{\partial x_{j}}+\frac{\partial\left(\sigma_{i j} u_{i}\right)}{\partial x_{j}}+\rho f_{i}^{e x t} u_{i}+S_{\text {energy }},
\end{gathered}
$$

with

$$
\begin{aligned}
\sigma_{i j} & =2 \mu\left[\frac{1}{2}\left(\frac{\partial u_{i}}{\partial x_{j}}+\frac{\partial u_{j}}{\partial x_{i}}\right)-\frac{1}{3} \frac{\partial u_{k}}{\partial x_{k}} \delta_{i j}\right], \\
J_{j} & =-\rho D \frac{\partial Y}{\partial x_{j}}, \quad q_{j}=-k \frac{\partial T}{\partial x_{j}}
\end{aligned}
$$

where $\rho, p, e$ and $u_{i}$ are the gas phase density, pressure, total energy and Eulerian velocity, respectively, and where $Y$ is the mass fraction of the evaporated dispersed phase. The heat and species mass diffusive flux vectors, $q_{j}$ and $J_{j}$ are give by Fourier's and Fick's law with $k$ and $D$ being the thermal conductivity and diffusivity, respectively. $\mu$ is the viscosity and $f_{i}^{e x t}$ stands for an external body force field.

The LES equations can be obtained by applying a spatial filter to the governing equations (2.1) to (2.4). The spatially filtered value of a function $\psi=\psi(\boldsymbol{x}, t)$ is defined as its convolution with a filter function, $G$, according to:

$$
\bar{\psi}(\boldsymbol{x})=\int_{\Omega} \psi(\boldsymbol{y}) G(\boldsymbol{x}-\boldsymbol{y} ; \Delta \boldsymbol{x}) \mathrm{d} \boldsymbol{y},
$$


where the integration is carried out over the entire domain, $\Omega$. The filter function, $G$, has a width $\Delta$ which may vary with position. In many problems, including the present case, it is desirable that $G$ be positive definite so that a subgrid probability density function may be defined. In situations where fluid density variations arise, the most straightforward method of accounting for these variations is through the introduction of density weighted (or Favre) filtering: $\tilde{\psi}=\overline{\rho \psi} / \bar{\rho}$. For the properties and limitations of the filtering approach see Germano $(1986,1992)$ and Piomelli (1999).

Applying density, weighted filtering to the governing equations then gives:

$$
\begin{gathered}
\frac{\partial \bar{\rho}}{\partial t}+\frac{\partial\left(\bar{\rho} \tilde{u}_{i}\right)}{\partial x_{i}}=\bar{S}_{\text {mass }}, \\
\frac{\partial\left(\bar{\rho} \tilde{u}_{i}\right)}{\partial t}+\frac{\partial \bar{\rho} \widetilde{u_{i} u_{j}}}{\partial x_{j}}=-\frac{\partial \bar{p}}{\partial x_{i}}+\frac{\partial \bar{\sigma}_{i j}}{\partial x_{j}}+\bar{\rho} \widetilde{f_{i}^{e x t}}+\bar{S}_{\text {mom }, i}, \\
\frac{\partial(\bar{\rho} \tilde{Y})}{\partial t}+\frac{\partial\left(\bar{\rho} \widetilde{Y u_{j}}\right)}{\partial x_{j}}=-\frac{\partial \overline{J_{j}}}{\partial x_{j}}+\bar{S}_{\text {species }}, \\
\frac{\partial(\bar{\rho} \tilde{e})}{\partial t}+\frac{\partial\left(\bar{\rho} \widetilde{e u_{j}}\right)}{\partial x_{j}}=-\frac{\partial \overline{p u_{j}}}{\partial x_{j}}-\frac{\partial \overline{q_{j}}}{\partial x_{j}}+\frac{\partial \overline{\sigma_{i j} u_{i}}}{\partial x_{j}}+\bar{\rho} \widetilde{f_{i}^{e x t}} u_{i}+\bar{S}_{\text {energy }},
\end{gathered}
$$

As a result of the filtering process, the quantities $\bar{\rho} \widetilde{u_{i} u_{j}}, \bar{\rho} \widetilde{Y u_{j}}$ and $\bar{\rho} \widetilde{e u_{j}}$ are unknown, and modelling - to account for subgrid scale contributions - is thus required. The filtered source terms can be interpreted as follows: if a particle, $p$, located at position $\xi^{(p)}$ provides a contribution $S^{(p)}$ to the sources for mass, momentum or energy then the total contribution of the particles within a filtering 'volume' to the source is just the volume average of the individual contributions, namely,

$$
\bar{S}(\boldsymbol{x})=\frac{1}{\Delta^{3}} \sum_{p} \int_{\Omega} S^{(p)} \delta\left(\boldsymbol{\xi}^{(p)}-\boldsymbol{y}\right) G(\boldsymbol{x}-\boldsymbol{y} ; \Delta(\boldsymbol{x})) \mathrm{d} \boldsymbol{y},
$$

where the summation is extended over all the particles present in the domain $\Omega$. In writing (2.6-2.9), it is has been assumed that the filter width is invariant in space. However, this is not the case in practical applications, where the need for different levels of spatial resolution across the solution domain requires the use of variable grid spacings and this results in a commutation error. This commutation error can be shown to be almost entirely dissipative in nature and negligible compared with dissipation of the SGS model.

\subsubsection{Subgrid models}

LES of inert flows have been studied extensively and the simplest model for the subgrid stress tensor,

$$
\tau_{i j} \equiv \bar{\rho} \widetilde{u_{i} u_{j}}-\bar{\rho} \tilde{u}_{i} \tilde{u}_{j}
$$

is the eddy viscosity model of Smagorinsky (1963)

$$
\tau_{i j}^{a}=-2 \mu_{s g s} \tilde{S}_{i j},
$$

where

$$
\mu_{s g s}=\bar{\rho}\left(C_{s} \bar{\Delta}\right)^{2}\|\tilde{S}\|, \quad \tilde{S}_{i j}=\frac{1}{2}\left(\frac{\partial \tilde{u}_{j}}{\partial x_{i}}+\frac{\partial \tilde{u}_{i}}{\partial x_{j}}\right), \quad\|\tilde{S}\|=\sqrt{2 \tilde{S}_{i j} \tilde{S}_{i j}},
$$

which linearly relates the anisotropic part of the subgrid stress tensor $\tau_{i j}^{a}$ to the filtered rate of strain tensor $\tilde{S}_{i j}$ via an eddy viscosity coefficient $\mu_{s g s}$ with a length 
scale $\Delta$ proportional to a measure of the grid spacing. For low-speed flows, such as considered here, the isotropic part of the stress can be adsorbed into the definition of the pressure to yield a pseudopressure, and modelling of this is not then required.

The length scale $\Delta$ is related to the filter width that, in LES practice, is invariably assumed proportional to some local measure of the mesh spacing. A commonly used measure is $\Delta=\left(\Delta_{x_{1}} \Delta_{x_{2}} \Delta_{x_{3}}\right)^{1 / 3}$ where $\Delta_{x_{1}}, \Delta_{x_{2}}$ and $\Delta_{x_{3}}$ are the physical grid spacings in the three coordinate directions, and this approach is employed in the present study. The model contains an adjustable parameter $C_{s}$, which must be specified. However, as many previous studies have demonstrated and, consistent with the ideas of Kolmogorov, results are found to be relatively insensitive to the value of $C_{s}$, providing, of course, that the turbulence Reynolds numbers are high and the largescale turbulent motions are adequately resolved. In circumstances where this is not the case, for example in transitional flows or in near-wall regions, viscous sublayer regions where adequate mesh resolution is often not possible, various dynamic calibration procedures offer some advantage (e.g. Germano 1992; Piomelli \& Liu 1995), and increase the generality of the model. In the present case, however, a single vale, $C_{s}=0.1$ has been selected. For the subgrid scalar flux $J_{k}$, it is usual to adopt a gradient model (see Schmidt \& Schumann 1989), of the form:

$$
J_{k} \equiv \bar{\rho} \widetilde{u_{k} Y}-\bar{\rho} \tilde{u}_{k} \tilde{Y}=-\frac{\mu_{s g s}}{\sigma_{s g s}} \frac{\partial \tilde{Y}}{\partial x_{k}},
$$

where $\sigma_{s g s}$ is the subgrid Schmidt number.

\subsection{Probabilistic treatment of the dispersed phase}

The spray equation developed by Williams $(1958,1985)$ represents one of the earliest attempts at a probabilistic description of sprays. Williams considered an ensemble of particles that could be characterized in terms of particle position, radius, velocity and temperature and derived a conservation equation for the particle distribution function. Further developments of the PDF approach to sprays includes the pioneering works of Buyevich (1971, 1972a,b) and Reeks (1980, 1991, 1992, 1993). The general application of the mathematical concepts developed in statistical physics to obtain a probabilistic description for particle-laden flows has been reviewed by Minier \& Peirano (2001) and Subramaniam $(2000,2001 a, b)$ have described the way in which Klimontovich and Liouville probability density definitions (Liboff 1998) can be re-adapted to the study of spray.

\subsubsection{Probabilistic treatment of a physical system}

A rigorous derivation of the probabilistic description of a physical system should be based on all the relevant microscopic variables, the values of which are given by the appropriate conservation equations. However, this is often difficult if not impossible and as an alternative a heuristic derivation can be pursued. Such an approach involves the selection of a restricted number of macroscopic variables, which are presumed sufficient to determine the physical state of the system. An evolution or transport equation for the joint probability density function of the variables selected can then be derived. In the heuristic philosophy, the effects of the microscopic interactions are replicated in the transport equation for the joint PDF of the macroscopic variables by the addition of stochastic terms, or generalized 'Langevin forces' (Risken 1984) to the Lagrangian deterministic differential equation governing each of the macroscopic variables. 
Let us suppose that the instantaneous state of a particle $j$ can be described by a set of $M$ independent macroscopic variables, $\boldsymbol{\Phi}^{(j)}(t)=\left\{\phi_{1}^{(j)}(t), \ldots, \phi_{M}^{(j)}(t)\right\}$. A population of $N$ particles can be characterized in state space using the fine-grained density function of the ensemble, which can be defined (see Lundgren 1967; Klimontovich 1969; Dopazo 1994), as:

$$
\mathscr{F}(\boldsymbol{\Psi} ; t)=\sum_{j=1}^{N} \prod_{i=1}^{M} \delta\left[\psi_{i}-\phi_{i}^{(j)}\right],
$$

where $\psi_{i}$ represents the phase or composition space for the quantity $\phi_{i}$ and where $\delta$ is the Dirac delta function.

From $\mathscr{F}(\boldsymbol{\Psi} ; t)$, it is possible to obtain a fine-grained probability density function, $P(\boldsymbol{\Psi} ; t)$. In order to use the term probability properly, its integration to unity and the concept of limiting value, which is approached as the number of realizations increases, must be ensured. The fine-grained probability density function is defined as the expectation of the fine-grained density. Expectation which, thanks to the central limit theorem, can be expressed as the limiting average over independent realizations of $\mathscr{F}$. If particles of the ensemble are also assumed to be independently distributed, the definition reduces to

$$
\mathscr{P}(\boldsymbol{\Psi} ; t)=\lim _{N \rightarrow \infty} \frac{1}{N} \sum_{j=1}^{N} \prod_{i=1}^{M} \delta\left[\psi_{i}-\phi_{i}^{(j)}\right]
$$

The hypothesis of independently distributed particles need not hold in order to be able to define a probability density function for the population, as demonstrated in Subramaniam (2000), however, such an assumption simplifies the definition.

By differentiating $\mathscr{P}$ with respect to time, following the motion in the state space $(\boldsymbol{\Psi} ; t)$ of a finite portion of the system, which is sufficiently small to be considered infinitesimal for the macroscopic variable gradients but large enough for the macroscopic variables to be defined, it is possible to obtain the conservation equation for the fine-grained probability density (for a complete derivation see Bini 2006):

$$
\frac{\partial}{\partial t} \mathscr{P}(\boldsymbol{\Psi} ; t)=-\sum_{i=1}^{M} \frac{\partial}{\partial \psi_{i}}\left\{E\left[\left.\dot{\psi}_{i}\right|_{\boldsymbol{\Psi}=\boldsymbol{\Phi}}\right] \mathscr{P}(\boldsymbol{\Psi} ; t)\right\},
$$

where $E\left[\left.\dot{\psi}_{i}\right|_{\boldsymbol{\Psi}=\boldsymbol{\Phi}}\right]$ represents the conditional rate of change of state space coordinate $\psi_{i}$ conditioned upon the particles being found at state $\boldsymbol{\Psi}=\boldsymbol{\Phi}$. In deriving (2.14), the differentiation of the generalized function $P$ must be considered in an integral manner, as the derivative of delta functions can only be defined when these are integrated, over a state space volume $\Omega$, taking their product with functions which are sufficiently regular on $\Omega$. The indicator function of the state space volume considered, $I_{\Omega}$, can be taken as such a function. This procedure is useful because it allows the use of the following identity: $E[X \delta(Z-Y)]=E(X \mid Y=Z) E[\delta(Z-Y)]=E(X \mid Y=Z) P(Z)$ which justifies (2.14). Equation (2.14) can be thought of as an exact, i.e. model free, but unclosed transport equation governing the probability density where the cause of the indetermination resides in the conditional rate of change of each state coordinate as functions of the state itself.

\subsubsection{The filtered PDF transport equation}

The solutions of any accurately modelled form of (2.14) for a population of particles transported in a turbulent flow provide information on the particle properties, e.g. 
velocity, over length and time scales ranging down to those comparable with and smaller than the Kolmogorov scales of the turbulent continuous phase. Because of the dependence of the particle properties on the velocity of the continuous phase, any moments derived from $\mathscr{P}(\boldsymbol{\Psi} ; t)$ will be smooth on the scale of the Kolmogorov lengths and times, Ijzermans, Hagmeijer \& van Langen (2007). The computational requirements for solving a suitably modelled form of (2.14) are thus likely to be comparable with those of a DNS of the continuous phase. Furthermore, the Lagrangian rates of change also depend on the full velocity spectrum of the continuous phase. Because in an LES of the continuous phase, no information is available concerning the flow variations on a scale smaller than the filter width, the knowledge of the spatial and temporal variations of the joint PDF of the variables describing the dispersed phase can be reliably obtained only to within the same range of scales, i.e. scales which are larger than the local filter kernel width. For this reason, a filtered PDF is defined. The filtered PDF is formed from the convolution of the joint PDF with the LES filter:

$$
\overline{\mathrm{P}}(\boldsymbol{\Psi} ; t))=\int_{\mathscr{D}} \mathscr{P}\left(\boldsymbol{\Psi} ; \boldsymbol{x}^{\prime}, t\right) G\left(\boldsymbol{x}-\boldsymbol{x}^{\prime}\right) \mathrm{d} \boldsymbol{x}^{\prime} .
$$

Such a convolution can also be viewed as the ideal framework for the definition of the degree of probability in a state space volume before the generalized function differentiation is carried out; rather than defining an indicator function in physical space, the LES filtering is considered (an indicator function, i.e. phase space binning, must still be considered for the remaining phase space variables). The transport equation for the filtered joint PDF can then be obtained in a manner which is directly analogous to the derivation of (2.14):

$$
\frac{\partial}{\partial t} \overline{\mathrm{P}}+\frac{\partial}{\partial \psi_{i}}\left[\mathrm{E}\left(\dot{\psi}_{i} \mid \boldsymbol{\Psi}=\boldsymbol{\Phi}\right) \overline{\mathrm{P}}\right],=0,
$$

where $\mathrm{E}(q \mid \boldsymbol{\Psi}=\boldsymbol{\Phi})$ represents the expected value of $q$ conditioned on $\boldsymbol{\Psi}$ being equal to $\boldsymbol{\Phi}$ anywhere within the filter volume. All moments resulting from the solution of (2.16) will be smooth at the filter-scale level.

\subsubsection{The LES spray equation}

To construct a statistical representation of the dispersed phase it is first necessary to select a set of macroscopic variables which are thought to be necessary and sufficient to describe the spray. Equation (2.16) can then be written for these variables. To describe the spray it is presumed here that the droplets are spherical so that their geometry can be defined by a single dimension $r$, the radius; this assumption relies on the hypothesis of small Weber number, typically $W e \ll 20$ (the Weber number provides a measure of the ratio of inertial and surface tension forces; when surface tension dominates, deformations and oscillations of the surface are minimized so that droplets remain essentially spherical in shape). Other necessary variables are the droplet velocity, $\boldsymbol{v}$ and the droplet temperature, $\theta$. In addition, it has been customary in the spray literature to introduce droplet number density, defined as the number of droplets present per unit volume. However such a variable is not a property of the dispersed phase alone and is thus not appropriate here. Instead a droplet number, $n$, defined as the number of droplets or particles is introduced as a stochastic variable. This is conserved and will only vary through particle coagulation (from 'collisions') and breakup and is uninfluenced by vaporization which acts to change the droplet radius and the gas phase density. It is thus presumed that the state of the spray 
can be determined uniquely from these four quantities so that the required PDF is $\overline{\mathrm{P}}_{s p r}(\boldsymbol{V}, \boldsymbol{R}, \Theta, \boldsymbol{N} ; \boldsymbol{x}, t)$, where $\{\boldsymbol{V}, \boldsymbol{R}, \Theta, \boldsymbol{N}\}$ is the 'phase' space for $\{\boldsymbol{v}, \boldsymbol{r}, \theta, \boldsymbol{n}\}$. The equation for this joint PDF can now be written:

$$
\frac{\partial \overline{\mathrm{P}}_{s p r}}{\partial t}+\nabla_{v} \cdot\left(\boldsymbol{a} \overline{\mathrm{P}}_{s p r}\right)+\frac{\partial\left(\dot{\mathscr{R}} \overline{\mathrm{P}}_{s p r}\right)}{\partial R}+\frac{\partial\left(\dot{\mathscr{T}} \overline{\mathrm{P}}_{s p r}\right)}{\partial \Theta}+\frac{\partial\left(\dot{\mathcal{N}} \overline{\mathrm{P}}_{s p r}\right)}{\partial N}=0,
$$

where $\boldsymbol{a}, \dot{\mathscr{R}}, \dot{\mathscr{T}}, \dot{\mathcal{N}}$ represent:

$$
\mathrm{E}\left(\frac{\mathrm{d} \psi_{k}}{\mathrm{~d} t} \mid \boldsymbol{\Psi}=\boldsymbol{\Phi}\right) \text { where } \boldsymbol{\Phi}=\boldsymbol{v}, r, \theta \text { and } n .
$$

Equation (2.17) is an exact unclosed hyperbolic partial differential equation for the joint PDF of the spray in which the filtered conditional Lagrangian rates are the cause of the indeterminacy.

\section{Modelling}

In this section, the models for the unclosed terms in the equations formulated in the previous sections are discussed. In the continuous-phase LES equations, models are required for the unknown subgrid scale, SGS stresses and fluxes and for the filtered source terms. The present work is devoted to dilute sprays, in which the gas-phase subgrid scale turbulent dissipation rate can be assumed essentially uninfluenced by the droplet motions. For this reason the SGS stresses and fluxes will be represented by the models for single-phase flow summarized in $\S 2.1 .1$. For the dilute sprays at present considered, the direct influence of droplet motions on the SGS stresses is likely to be negligible, particularly when compared with the influence of SGS fluctuations on the unclosed terms of (2.17).

The unclosed terms appearing in (2.17) can be modelled following the heuristic procedure previously mentioned. Mathematically, this approach consists of adding a stochastic contribution to each of the deterministic Lagrangian differential equations governing the evolution of the macroscopic variables. This approach is consistent with LES concepts: in LES, the filtered quantities are simulated directly with the subfilter contributions, arising from the nonlinearity of the governing equations, being modelled. If the Lagrangian differential equations for the rate of change of each macroscopic liquid variable are evaluated in terms of filtered flow-field quantities plus a stochastic contribution, then this may be viewed as a direct simulation of the behaviour of the filtered macroscopic variables with the stochastic contribution representing the (nonlinear) influence of the sub-filter scales. Each of the unclosed terms, equation (2.17), can thus be split in the following way:

$$
\mathrm{E}\left(\frac{\mathrm{d} \psi_{k}}{\mathrm{~d} t} \mid \boldsymbol{\Psi}=\boldsymbol{\Phi}\right)=f\left(\psi_{k}, \tilde{\phi}_{g a s} ; t\right)+\chi_{k},
$$

where the first term on the right-hand side of (3.1) represents a deterministic contribution to the Lagrangian rate of change evaluated in terms of the filtered gas phase properties $\tilde{\phi}_{g a s}$ etc. and where:

$$
\chi_{k}=\mathrm{E}\left(\frac{\mathrm{d} \psi_{k}}{\mathrm{~d} t} \mid \boldsymbol{\Psi}=\boldsymbol{\Phi}\right)-f\left(\psi_{k}, \tilde{\phi}_{g a s} ; t\right),
$$

represents the effects of the SGS fluctuations on the filtered conditional Lagrangian rates of change. The decomposition, (3.1), can be viewed as representing a natural extension of that adopted for defining the subgrid-scale stresses and fluxes. As 
discussed in earlier sections, the neglect of SGS fluctuations can lead to significant error and subgrid-scale models are thus required for $\chi_{k}$.

The formulation of stochastic models has been discussed in Miller \& Bellan (2000) and Okong'o \& Bellan (2004), where it was proposed that the random contribution of a flow variable $\phi$ be related to a realization of a random variable having a standard deviation proportional to the standard deviation of the SGS fluctuations of that variable, i.e. $\sigma_{s g s}^{2}=\left(\widetilde{\phi^{2}}-\tilde{\phi}^{2}\right)$. However, such an approach can only be properly applied to scalar quantities and its use for vector variables, e.g. the gas velocity $u_{i}$, is in conflict with the basic transformation properties of a vector, as is discussed in Appendix A. The present work focuses attention on particle dispersion and particle-turbulence interaction in a shear flow, and droplet vaporization and breakup are negligible in the cases presently considered. The droplets are 'dilute' and breakup processes are unimportant and are therefore neglected. As a consequence, models for the rates of change of particle size, temperature and breakup and coagulation, $\dot{\mathscr{R}}, \dot{\mathscr{T}}, \dot{\mathscr{N}}$ are not required here. However, the general formulation, (2.17), does provide a rational framework for the incorporation of these latter effects and expressions capturing sub-filter effects upon vaporization and breakup have been proposed, for example, in Bini (2006).

\subsection{Rate of change for the droplet velocity}

The model for the particle acceleration, $\boldsymbol{a}$, is directly responsible for particle dispersion in a turbulent flow field. The deterministic part is taken to be equal to the first term of the Maxey \& Riley (1983) formulation for the force per particle mass:

$$
\boldsymbol{a}=\frac{\mathrm{d} \boldsymbol{v}_{p}}{\mathrm{~d} t}=\frac{\overline{\boldsymbol{u}}-\boldsymbol{v}_{p}}{\tau_{p}}+\chi,
$$

where $\boldsymbol{v}_{p}$ is the particle velocity, $\overline{\boldsymbol{u}}$ is the known filtered flow velocity at the particle position and $\tau_{p}$ is the particle response time:

$$
\tau_{p}^{-1}=\frac{3}{8} C_{D} \frac{\left|\overline{\boldsymbol{u}}-\boldsymbol{v}_{p}\right|}{r_{p}}
$$

where $r_{p}$ is the particle radius and $C_{D}$ the particle drag coefficient which can be accounted, for example, using the drag law of Yuen-Chen, (Sirignano 1999).

The random contribution is deemed to have the following general properties: it can be integrated in time such that the increment $\mathrm{d} \boldsymbol{v}_{p}$ occurs over a time interval $\mathrm{d} t$, it must possess the properties of random vectors (see Yaglom 1957), and it must represent the 'diffusion' of particles owing to the unresolved fluctuations. For these reasons, a multidimensional Wiener process can be selected. This satisfies the first two properties exactly and has been widely used for diffusion-like processes. It corresponds to a closed solution of the Fokker-Plank equation when there is no drift. A time-discretized Wiener process has the form:

$$
W\left(t_{n}\right)=\delta t^{1 / 2} \sum_{j=1}^{n} \xi_{j},
$$

where $t_{n}=n \delta t$ and $\xi$ is a random variable with zero mean and a variance of unity (the extension to processes having non-uniform time increment does not present additional complications). The term $\chi \mathrm{d} t$ is thus written as a Wiener vector process pre-multiplied by a diffusion coefficient matrix which serves to adjust the diffusion 
and to relate it to the turbulence intensity of the gas phase.

$$
\mathrm{d} \boldsymbol{v}_{p}=\tau_{p}^{-1}(\overline{\boldsymbol{u}}-\boldsymbol{v}) \mathrm{d} t+\boldsymbol{B} \cdot \mathrm{d} \boldsymbol{W}
$$

In the present work, the diffusion process is taken to be isotropic and the diffusion matrix thus has the simplified form: $\boldsymbol{B}=b \delta_{i j}$. The extension to a non-isotropic coefficient matrix is discussed in Appendix A. The use of the Weiner process in representing the influence of unresolved velocity fluctuations has the consequence that acceleration cannot be defined in the sense of classical calculus. However, the acceleration PDF can be defined in terms of $\delta \boldsymbol{v} / \delta t$ for a small but finite $\delta t$ with the PDF then being obtained by writing $\delta \boldsymbol{v}$ as an Ito integral of $\mathrm{d} \boldsymbol{W}$, which is well-defined and convergent (Gardiner 2002).

Thomson (1987) showed, using only the Markov and continuity hypothesis, how the Lagrangian velocity in a single-phase flow can be represented by a generalized Langevin equation in which the stochastic dispersion is written as:

$$
\chi_{j}=\sqrt{C \epsilon} \mathrm{d} W_{j}
$$

where $\epsilon$ is the mean dissipation rate of turbulence kinetic energy and $C$ is Kolmogorov's Lagrangian velocity structure function constant.

In the present case of material particles, the diffusion coefficient, $b$ may be expected to depend on the time and length scales of SGS velocity fluctuation of the continuous phase and the particle properties. If the SGS turbulence kinetic energy, $k_{s g s}$ is used to characterize the velocity fluctuations then we may write:

$$
b=\sqrt{C_{0} \frac{k_{s g s}}{\tau_{t}}},
$$

where $C_{0}$ is a model constant which, in the present work, is assigned a value of unity and $\tau_{t}$ is a time scale which affects the rate of interaction between the particle and turbulence dynamics.

Now it is known - see the earlier discussion - that particle accelerations at the small scales exhibit a probability density function which deviates strongly from Gaussianity. By suitable choices of $\tau_{t}$, Bini \& Jones (2007) have shown how a family of stochastic processes can be generated that account for this. If $\tau_{t}$ is made to depend on the random variable $\boldsymbol{v}_{p}$ itself then, depending on the dependence, different asymptotic forms for the PDF of particle velocity increment can be obtained. Bini \& Jones (2007) constructed a stochastic process which is able to reproduce the desired behaviour of the PDF of particle acceleration without over-imposing the PDF shape and which correctly attributes high levels of probability to extreme acceleration events. A time scale given by:

$$
\tau_{t}=\frac{\tau_{p}^{2 \alpha}}{\left(\Delta / \sqrt{k_{s g s}}\right)^{2 \alpha-1}},
$$

with $\alpha=0.8$, was shown to reproduce the high values of kurtosis and PDFs observed experimentally.

The time scales $\tau_{t}=\Delta^{1 / 3} /\left|\boldsymbol{v}_{p}\right|$ and $\tau_{t}=\Delta^{1 / 3} / \sqrt{k_{s g s}}$ may be considered as alternatives. These latter two scales are related to the time taken for a particle to cross a notional 'unresolved eddy'. Following the reasoning of Bini \& Jones (2007), it is to be noted that whilst the former time scale will result in a leptokurtic behaviour of the small-scale acceleration PDF, because of the appearance of term $\left|\boldsymbol{v}_{p}\right|^{1 / 2}$ in the denominator, the latter choice will give rise to particle acceleration PDFs that are Gaussian. 


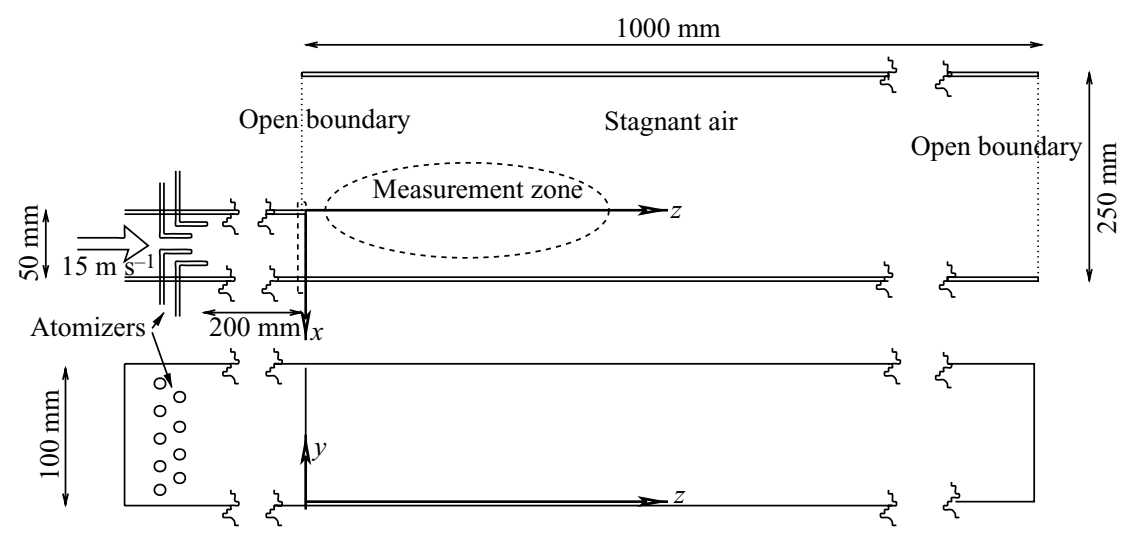

FIGURE 1. Experimental apparatus and physical domain.

\subsection{Modelling of the filtered source terms}

The source terms appearing in the gas phase equations can be evaluated by interpreting them, (2.10), as the volume average of the source contributions of each of the stochastic particles lying within the filtering volume. In the context of a large-eddy simulation in which the finite-volume method is used to discretize the equations this is equivalent to averaging over the cell volume. The source terms can thus be written:

$$
\bar{S}=\frac{1}{\Delta^{3}} \sum_{\alpha=1}^{n^{*}} S^{(\alpha)},
$$

where $n^{*}$ is the number of stochastic particles present in the cell volume considered and $S^{(\alpha)}$ is the source term arising from droplet $\alpha$. In the present case, the relevant term for the LES equations is:

$$
S_{m o m, i}^{(\alpha)}=-\left(\frac{\mathrm{d} m v_{i}}{\mathrm{~d} t}\right)^{(\alpha)}
$$

\section{Flow simulated: a droplet-laden spatially developing mixing layer}

In order to demonstrate the capabilities of the filtered PDF approach and the effectiveness of the proposed models, in this section the results of computations are presented for the spatially developing mixing layer studied experimentally by Lazaro \& Lasheras $(1989,1992 a, b)$. The experimental apparatus and physical domain are shown schematically in figure 1 . The test section comprises a rectangular duct of cross-section $250 \mathrm{~mm} \times 100 \mathrm{~mm}$ and length $1000 \mathrm{~mm}$. Both ends of the duct are open. The liquid droplets (the dispersed phase) are injected upstream of the test section by means of a series of atomizers into a channel where the droplets and air mix and the spray homogenizes. At the exit of the channel the droplet-laden stream exits into the duct and a mixing layer forms downstream of the upper channel wall. The operating conditions are such that the spray is dilute and that droplet evaporation effects are negligible. Gravitational sedimentation effects are also negligible in the region of the mixing layer where the measurements were conducted. The computational domain corresponds to the region $-200<x<+50 \mathrm{~mm}$, $0<y<+100 \mathrm{~mm}$ and $0<z<+1000 \mathrm{~mm}$. The measured characteristics of the dropletladen gas stream together with many features about the dispersed phase have been measured at a plane corresponding to the nominal origin of the mixing layer at $z=0$, 
(a)

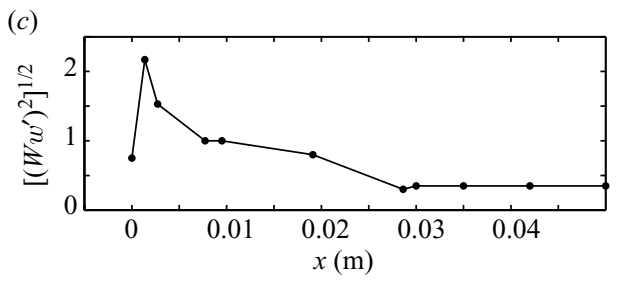

(b)

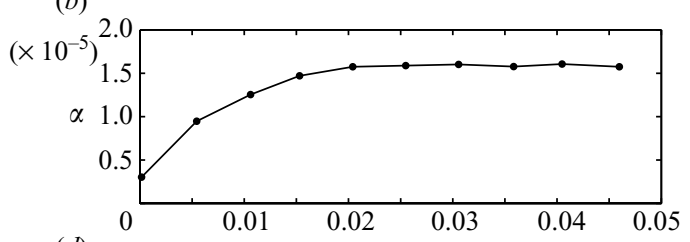

(4)

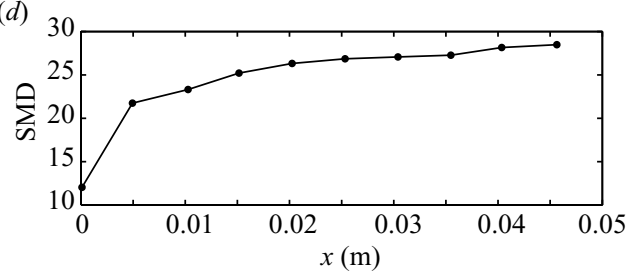

Figure 2. Inflow conditions: cross-stream profiles of the first two moments of $w(a, c)$, cross-stream profiles of liquid to air concentration $\alpha(b)$ and SMD $(d)$.

from here on referred to as the inflow plane. These measured properties constitute the inflow conditions for the presented calculations and are shown in figure 2. The Reynolds number based on the boundary-layer thickness at the inflow plane is 10000 . The inflow data available, though detailed, is not sufficient to completely specify the inflow joint probability density function of the dispersed phase that is required for solution of the spray equation (2.17). In particular, the PDF of the particle diameter is available at only a few inflow positions and there is no information available on the incoming droplet velocity distribution at the inflow plane.

In many previous experimental studies of droplet-laden flows it has often been argued that, when the spray has reached a good degree of homogeneity, the velocity statistics of the continuous (in this case air) and dispersed phases can be considered to be closely similar. Based on this - because in the present case the atomizers are located relatively far upstream from the inflow plane - one method for determining the entering particle velocity PDF would be to sample from velocity distribution functions with moments, i.e. the mean velocity and Reynolds stresses, corresponding to the measured values of the inflow velocity. However, trial computations have shown that such an assumption is unsuitable in the present case. Turbulent boundary layers develop along the channel walls and are clearly visible from the measured mean and turbulence profiles of the gas phase (figure 2). If the boundary-layer profiles of mean velocity and turbulence intensities are used as a basis for estimating droplet velocities at the inflow plane then, near the channel wall, a significant number will enter the domain with a very small velocity. On entering the domain, these particles will continue to move at a slow speed with a consequence that a maximum in the liquid concentration arises close to the inflow plane, a feature not evident in the experiments.

Righetti \& Romano (2004) have questioned the validity of the assumption that the velocity statistics of the continuous and dispersed phases are similar, particularly the assumption of 'no slip' between the two phases. Supported by measurements in droplet-laden channel flows, they concluded that the mean velocities of the particles can be very different from those of the gas flow, though it is reasonable to assume that they have the same variance. Kulick, Fessler \& Eaton (1994) attribute the differences in the particle and fluid velocities mainly to inertia effects: high-momentum particles tend to retain their velocity when moving towards a solid surface. However, it remains 


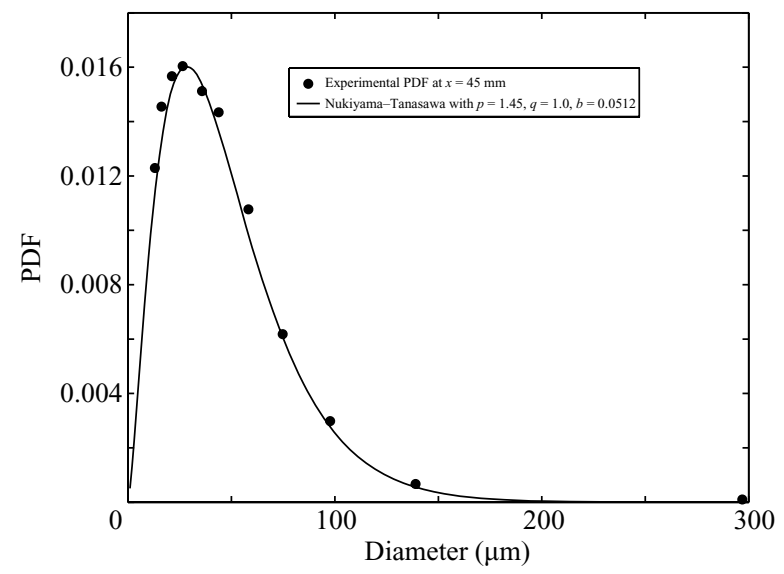

FIGURE 3. Inflow conditions: $\operatorname{PDF}(D \mid(x=0.45, z=0))$, experimental findings $(\bullet)$, prescribed functional form of the Nukiyama-Tanasawa family (line).

unclear how the difference between the streamwise velocities depends on the particle sizes and on the distance from the wall and how they are related to turbulence dynamics in the wall region.

In the present work, a relatively simple strategy has been adopted for estimating the velocities of the particle entering close to the wall. First, it is presumed that particles injected far away from the wall region, i.e. outside the boundary layer, are effectively carried by the gas phase with, on average, no-slip velocity. For particles injected in the near-wall region, the particle velocity at the injection point, $v_{a}(d)$ is estimated and the velocity at the inlet plane is then computed using the deterministic part of (3.2) only, under the assumption that the streamwise gas phase velocity is zero. This is done for droplets of various diameters, $d$, so that, at the inlet plane and in the vicinity of the wall, the droplet velocity distribution, $v_{z}(d)$ is determined as a function of diameter.

The problem regarding the interpolation of the droplet diameter PDF measured at discrete $x$-positions to other inflow locations has been solved as follows: the data available seem to be well represented by the Nukiyama-Tanasawa distribution function (Lefebvre 1989) which depends on the three independent parameters, $p, b, g$ :

$$
P(D)=a D^{p} \mathrm{e}^{-(b D)^{g}} .
$$

By a suitable choice of the parameters $p, b, g$ it has been found that this distribution can be made to fit all of the measured inflow data accurately. It is then used to provide the required inflow conditions; the parameters $p$ and $q$ are maintained constant while $b$ is varied to reproduce the measured profile of Sauter mean diameter, SMD; a typical result is shown in figure 3.

\subsection{Computational details}

The in-house LES code BOFFIN has been used for the computations to be presented in the following sections. The code employs Cartesian velocity components with a colocated variable storage arrangement. It is based on a fully implicit low-Mach-number formulation and is second-order accurate in space and time. For the momentum equation convection terms, an energy conserving discretization scheme is used and the advancement in time is performed with an implicit method of the Gear family with variable time step. The code has been applied previously to the LES of a wide range 
of flows including plane and round jets in a crossflow (Jones \& Wille 1996a,b), fully developed channel (Wille 1997), and pipe flows (Steinstrasser 1994), flow over a swept fence (di Mare \& Jones 2003) and non-swirling and swirling jet flames (Branley \& Jones 1999, 2001). Further details of the method may be found in Wille (1997) Branley (1999) and di Mare (2002). In all the calculations presented here, the SmagorinskyLilly model, with a constant equal to 0.1 , has been adopted for the SGS stress, and the source terms, equation (3.2), have been incorporated. The modelled Lagrangian form of the spray PDF equation (2.17), has been solved using a stochastic particle method in conjunction with a finite-difference scheme based on the method of fractional steps (Yanenko 1971). Each time step is decomposed into a series of (unphysical) sub-steps in which only one of the state space variables of (2.17) is allowed to vary using the appropriate modelled Lagrangian rate of change; the scheme used for the numerical integration of the SDEs was a simple Euler discretization (Kloeden \& Platen 1992). Each stochastic particle has a velocity, $v_{i}$, a number, $n$, a radius, $r$ and a temperature $\theta$ and the tracking of the particles in phase space is conducted in a manner directly analogous to that used in tracking physical particles. In order to compute statistical properties of the spray in each LES 'cell', particle trajectories were computed over a sufficiently long time interval with particles being allowed to leave the domain at the exit plane and new particles being added, with properties corresponding to the specified profiles, at the inflow plane. The properties of the dispersed and gas phase are updated once every time step: the updated gas flow field is used in (2.17).

All of the computations presented were carried out using a grid having $81 \times 21 \times 251$ nodes in the $x, y$ and $z$ directions, respectively. At the inflow plane $z=0$ and for $x \geqslant 0$ inflow conditions were imposed whereas for $x<0$ simple Neumann boundary conditions were applied. At the lateral boundaries-free-slip wall conditions were adopted. At the outflow boundary, $z=1000 \mathrm{~mm}$, a convective outflow condition is applied:

$$
\frac{\partial u_{i}}{\partial t}+U_{c} \frac{\partial u_{i}}{\partial n}=0,
$$

where $n$ is the distance normal to the boundary and $U_{c}$ is a constant convective velocity whose value is determined in terms of the overall mass inflow rate.

The flow entering the inflow plane comprises a fully developed turbulent boundary layer and the specification of realistic turbulent inflow conditions is crucial for the accurate simulation of the downstream evolution of the mixing layer. The problem of providing a realistic and reliable representation of inflow turbulence is a well-known problem in both LES and DNS, which has limited the study of spatially developing flow; often the problem can be overcome by the use of scaled periodic conditions applied as inflow boundary profiles. However, the specification of turbulent inflow conditions cannot be avoided in the present case. For this reason, the proposed digital filter methodology of Klein, Sadiki \& Janicka (2003) and di Mare et al. (2006) is used to generate turbulent inflow profiles. A digital filter is applied to maps of random numbers to construct turbulence data which are spatially correlated (the method also allows the generation of temporally correlated data). The approach involves a prescribed shape for the two-point correlation tensor in terms of an integral length scale, selected to correspond to the flow being considered. The correlated maps obtained by the digital filtering algorithm are used as instantaneous inflow velocity profiles that reproduce, when averaged, the specified mean and Reynolds stress profiles. The key steps of the method are outlined in Appendix B. 


\section{Results}

The results of the computations are presented in a sequence corresponding to that adopted in the original papers describing the experimental data.

\subsection{Gas mixing-layer characterization}

Measurements of the Reynolds averaged mean, $\bar{w}_{g}$, and r.m.s., $\bar{w}_{g}^{\prime}$, longitudinal gas velocity cross-stream profiles were performed at equally spaced downstream stations spanning the first $260 \mathrm{~mm}$ of the domain. From these profiles, two quantities were used in order to define the lateral spread of the mixing layer: the momentum thickness, $\theta(z)$, and the $0.1-0.9$ level thickness, $\delta_{08}(z)$. These are defined, at every $z$ location, as:

$$
\begin{aligned}
\delta_{08} & =x\left(\bar{w} / \bar{w}_{\infty}=0.9\right)-x\left(\bar{w} / \bar{w}_{\infty}=0.1\right), \\
\delta_{m} & =\int_{-\infty}^{\infty} \frac{\bar{w}}{\bar{w}_{\infty}}\left(1-\frac{\bar{w}}{\bar{w}_{\infty}}\right) \mathrm{d} x,
\end{aligned}
$$

where $\bar{w}_{\infty}$ is the value in the fast-moving particle-laden side, at the particular $z$ location considered. Several computations have been carried out to assess the dependency of the downstream spreading rate on the estimated integral length scale of the prescribed inflow turbulence. In particular, the results of three computations are presented (figure 4) in which only the length scale required by the inflow turbulence generator is varied. The results clearly show that correlated turbulent inflow profiles are required if the spreading rates are to be correctly reproduced. With an integral length scale of $2.5 \mathrm{~mm}$, a value roughly equal to a quarter of the boundary-layer thickness at the inflow, the downstream spreading rate of the two mixing-layer thicknesses is accurately reproduced. This value of the prescribed length scale is used in all the comparisons presented below. Additional confirmation of the accuracy of the solution achieved can be gained with the comparison of the computed and experimental profiles of the longitudinal mean velocity and turbulence intensity. Following Lazaro \& Lasheras, the measured and computed profiles are presented in similarity coordinates, $(x, z) \rightarrow(\eta, z)$ where

$$
\eta(x, z)=\frac{\left(x-x_{\bar{w}=\bar{w}_{\infty} / 2}(z)\right)}{\delta_{m}(z)} .
$$

Figure 5 shows comparison between measured and predicted profiles at all measured downstream locations, i.e. $0 \leqslant z \leqslant 280 \mathrm{~mm}$. For the mean profiles, the level of agreement achieved is excellent. The maximum levels and profiles of turbulence intensity are also reproduced well with the exception of the region $\eta \leqslant-4$ in the stagnant-air side of the mixing layer. The high levels of intensity predicted were found to arise regardless of the inflow conditions and a detailed examination of an animation of the simulation suggests that they originate from the recirculation (and backflow) relatively weak - evident in the simulations in the 'stagnant' air outside of the mixing layer. Whether or not such a recirculation is present in the experiment is uncertain. It has been shown (Scheffer, Hartmann \& Dibble 1987) that the measurements of velocity in jets can be affected by counterflows in zones outside the jet. Finally, for the gas phase, the computed and experimentally measured power spectral density for the $z$-component velocity sampled by a probe located at $\left(x_{p}, z_{p}\right)=(5 \mathrm{~mm}, 178 \mathrm{~mm})$ is shown in figure 6. The numerical signal has been acquired every time step, i.e. at intervals of around $10^{-5} \mathrm{~s}$, corresponding to sampling at a frequency of approximately $100 \mathrm{kHz}$. On the other hand, the smallest resolved turbulent scales are of size of the order of the filter width, $\approx 1-2 \mathrm{~mm}$, and have a frequency of around $700 \mathrm{~Hz}$. The difference between the spatial resolution and the sampling-time interval is responsible 

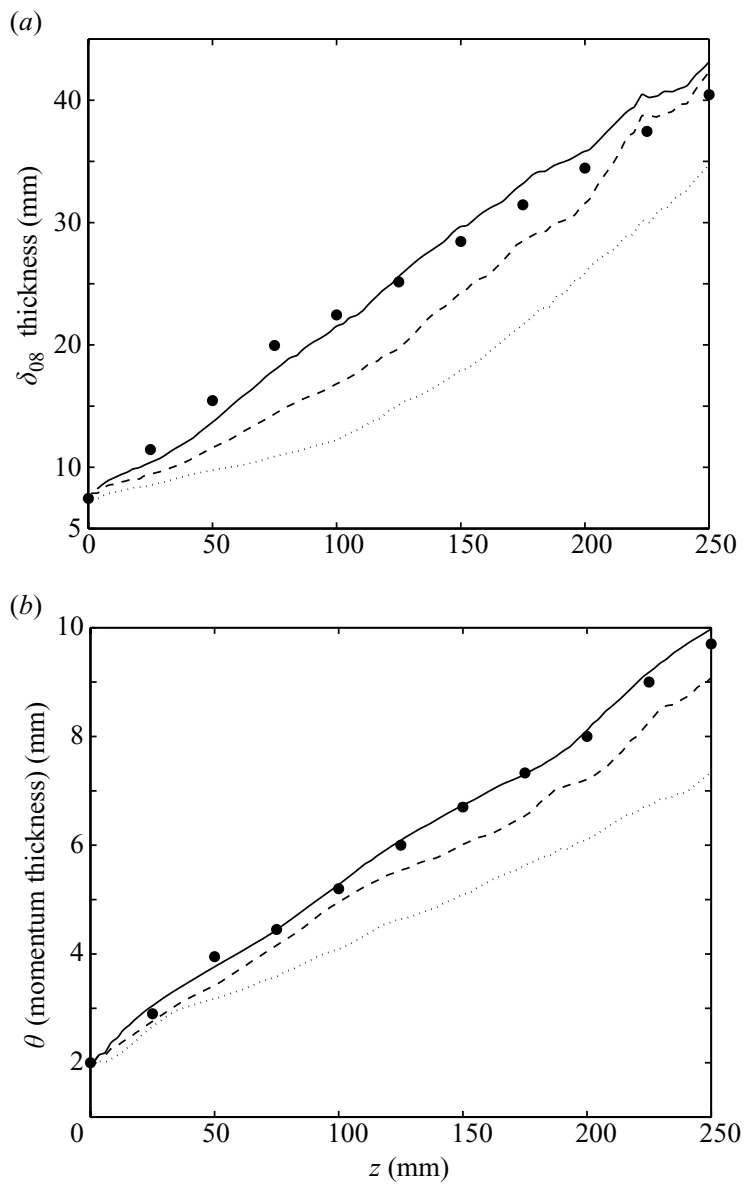

FIGURE 4. Downstream spreading of longitudinal momentum: $(a) \delta_{08} ;(b) \theta$. Experiment $(\bullet)$; computed with no digital turbulence (dotted line); computed with digital turbulence having filter width $L=1.5 \mathrm{~mm}$ (dashed line); computed with digital turbulence having filter width $L=2.5 \mathrm{~mm}$ (solid line).
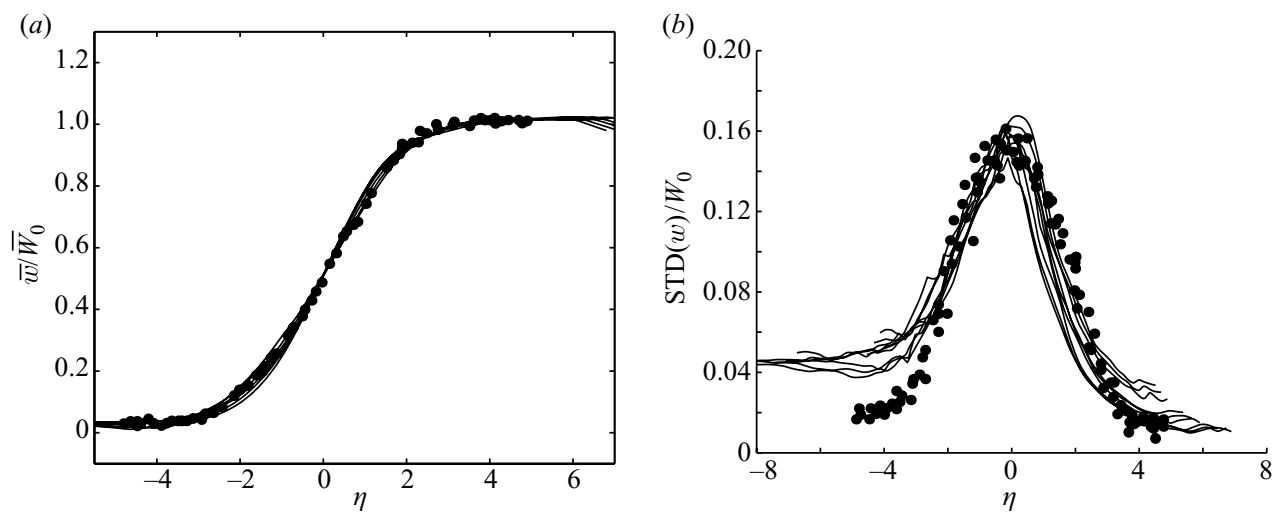

FIGURE 5. Downstream profiles in similarity coordinates between $z=50$ and $250 \mathrm{~mm}$ : $(a)$ normalized mean profiles; $(b)$ normalized standard deviation profiles. Computed (solid lines), experiment $(\bullet)$. 


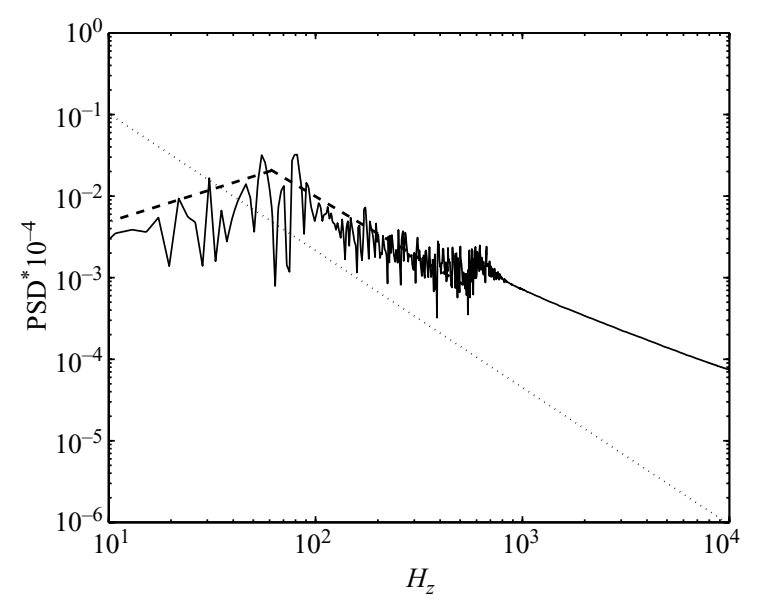

FIGURE 6. Fourier transform of the autocorrelation coefficient of the longitudinal velocity sampled at $(x, z)=(5 \mathrm{~mm}, 178 \mathrm{~mm})$. Computed (solid line); qualitative behaviour of the experimental (dashed line); $-5 / 3$ (dotted line).

of the straight line appearing in the spectrum for frequencies higher then the spatially resolved ones. From the figure, it is evident that the LES filter is correctly applied in the inertial range region and, as suggested by Lazaro \& Lasheras, the absence in the spectrum of a marked maximum can be taken as an indicator of important three-dimensional effects. Structures involving Kelvin-Helmholtz instabilities, whose effects might be expected to appear in the spectrum at a particular frequency, undergo distortion and further instability in the normal direction, making a definite maximum more difficult to identify. A well-defined maximum at $\approx 60 \mathrm{~Hz}$ has been identified in 'two-dimensional LES' computations, the results of which are not reported here.

\subsection{Droplet dispersion characteristics}

In the experimental work, the local liquid concentration, $\alpha$, defined as the ratio of the volume occupied by the liquid and the volume occupied by the gas is used to characterize the dispersed phase. Before attempting definitive comparisons between the LES results and measurements, it is clearly important to demonstrate that inflow conditions can be estimated, using the method described above, without them having critical effects on the calculations. The effects of the streamwise droplet inflow velocities and a realistic method of specifying them has already been discussed above and now the effects of the cross-stream droplet inflow velocity are examined. In figure 7, the normalized profiles of the mean liquid concentration, at two different downstream locations, $z=102 \mathrm{~mm}$ and $z=254 \mathrm{~mm}$, are presented for three computations identical in all but the distribution from which the droplet inflow cross-stream, i.e. $x$-component, velocities were sampled. In all three computations, the droplet response time-SGS dispersion model was used. They correspond to: Case A in which the cross-stream droplet velocity, $u$, is obtained by random sampling at all $(x, y)$ from a Gaussian distribution having zero mean and unit standard deviation; Case B where $u$ is sampled from a Gaussian distribution with a zero mean and a standard deviation corresponding to that of the measured gas phase streamwise velocity, (figure 2); Case $\mathrm{C}$ is identical to Case $\mathrm{B}$ except that the standard deviation is reduced by $40 \%$. Cases $\mathrm{B}$ and $\mathrm{C}$ rely on the previously mentioned proposition that the second moments of the dispersed and continuous phase velocity PDFs can be 

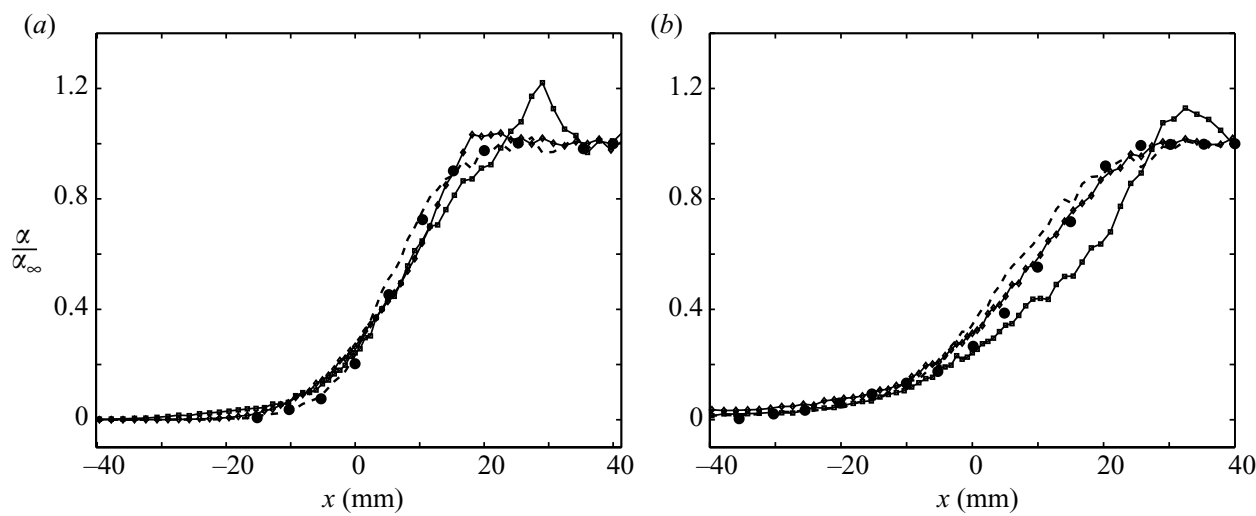

Figure 7. Normalized mean liquid to air concentration, effects of the inflow droplet cross-stream velocity: profiles at $(a) z=102 \mathrm{~mm},(b) z=254 \mathrm{~mm}$. Experiment $(\bullet)$, computed with droplet velocity sampled from velocity distribution having zero mean and standard deviation: uniform and equal to $1 \mathrm{~m} \mathrm{~s}^{-1}(--)$, with the same profile and value of $w$ gas $(\boldsymbol{\square})$, with the same profile of $w$ gas but intensity rescaled of $60 \%(\diamond)$.

assumed to be similar. Case B is based on the observation that, in turbulent channel flows, the profile of the standard deviation of the velocity component normal to the wall is usually found to be of the same qualitative shape as that of the streamwise velocity, but with a magnitude that is smaller by around about 50 to $70 \%$. Comparing the three, it appears that there is little difference between the profiles corresponding to Cases A and C while the higher intensity of Case B causes a change in the predicted physics with particles being more likely to be captured by the vortical structures developing towards the edge of the mixing layer. Such structures can be identified by the local maxima and minima in the iso-contour map of the pressure field (figure 8) and can be seen in the mushroom-like structures in the 'mixture fraction' contours also shown in figure 8. In Case B, stochastic particles are found to be clustered around the edge of the vortical structures and give rise to the unrealistic maxima evident in figure 7. The computed evolution of the profiles of the normalized mean liquid to air concentration with downstream distance are shown in figure 9. The effect of 'diffusion' of the liquid droplets is clearly evident. It results from both the resolved and unresolved droplet transport, but is mainly dependent on the gas phase turbulencedroplet interactions. In order to shed more light on droplet transport, a comparison of the mean liquid concentration cross-stream profiles at two different downstream locations, $z=102 \mathrm{~mm}$ and $z=254 \mathrm{~mm}$ are shown in figure 10 . In addition to the measured profiles, the results of three computations are plotted. These correspond to the cases (i) without the SGS droplet dispersion model, (ii) with the dispersion model, (3.8). and (iii) (3.7), but with $\tau_{t}=\Delta^{1 / 3} /\left|\boldsymbol{v}_{p}\right|$. In the latter two cases the value of the constant, $C_{0}$ is set to unity. (The performance of the model, (3.8), has been shown, elsewhere, to be relatively insensitive to the value of the constant $C_{0}$ in the range $0.5<C_{0}<1.5$ ). At the first downstream location, the difference between the results of the three models is small, though some trends are suggested. With no SGS dispersion, particle 'diffusion' is insufficient whereas with $\tau_{t}=\Delta /\left|\boldsymbol{v}_{p}\right|$ it is too large. Such a trend is much more clearly visible at the furthermost downstream location. Here, with no SGS dispersion, it is clear that the profile is insufficiently 'diffuse' and the agreement with the measured profile is poor. In the case of the SGS dispersion model, (3.8), the predicted profile is in good agreement with the measurements. In contrast, when the 

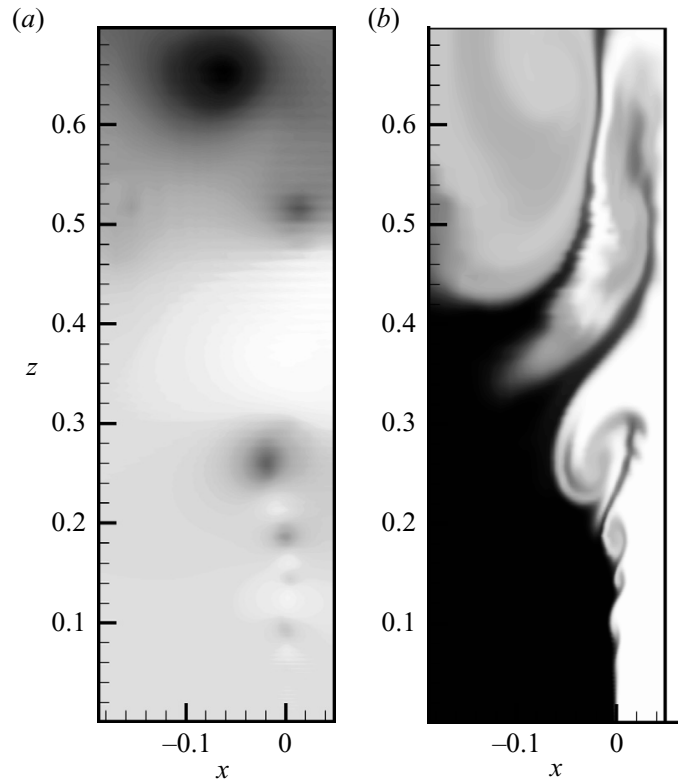

FIGURE 8. Gas phase: isocontour maps $(a)$ for the pressure and $(b)$ for the mixing of a passive scalar transported by the droplet-laden stream.

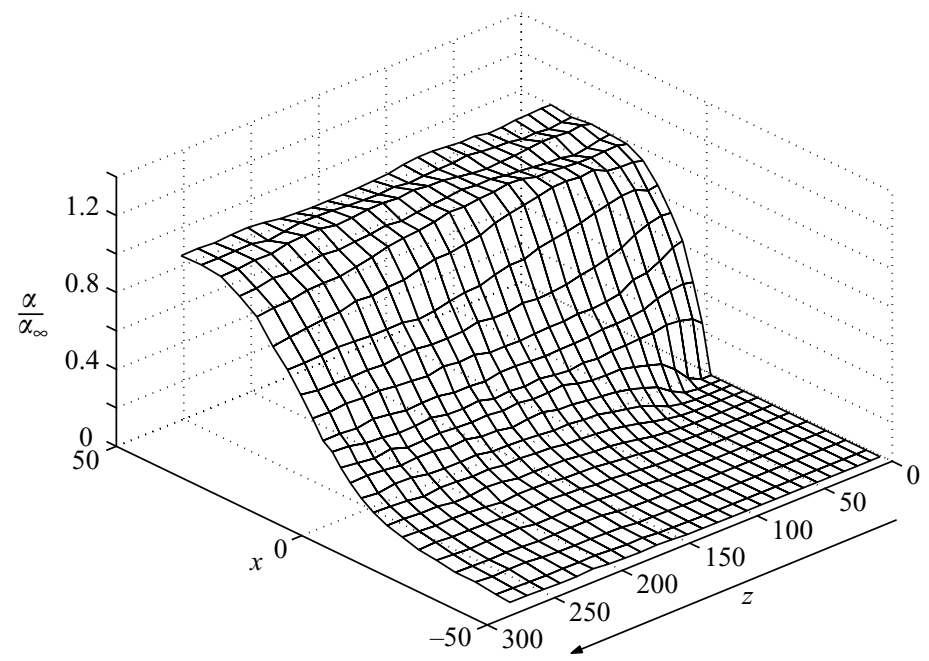

FIGURE 9. Computed Normalised mean liquid to air concentration, $\alpha / \alpha_{\infty}, \alpha=V_{L i q} / V_{a i r}$.

'filter crossing time', $\Delta^{1 / 3} /\left|\boldsymbol{v}_{p}\right|$ is used, the results obtained are again poor. Typically, the particle response time lies within the range between $10^{-4}$ and $10^{-5} \mathrm{~s}$ whereas the 'filter crossing time' is of the order of $10^{-3}$ to $10^{-4} \mathrm{~s}$. In addition to producing poor results, the use of the 'filter crossing time' is in any case of questionable validity as, in general, $\Delta^{1 / 3} /\left|\boldsymbol{v}_{p}\right| \rightarrow \infty$ as $\left|\boldsymbol{v}_{p}\right| \rightarrow 0$. For the computation using the SGS dispersion model, the mean downstream spreading of the liquid phase is compared, in figure 11, with the experimentally measured spreading rate. This spreading rate is based on a characteristic thickness, defined as the distance between the locations at which the mean liquid to air concentration is $90 \%$ and $10 \%$, similar to that used for the gas 

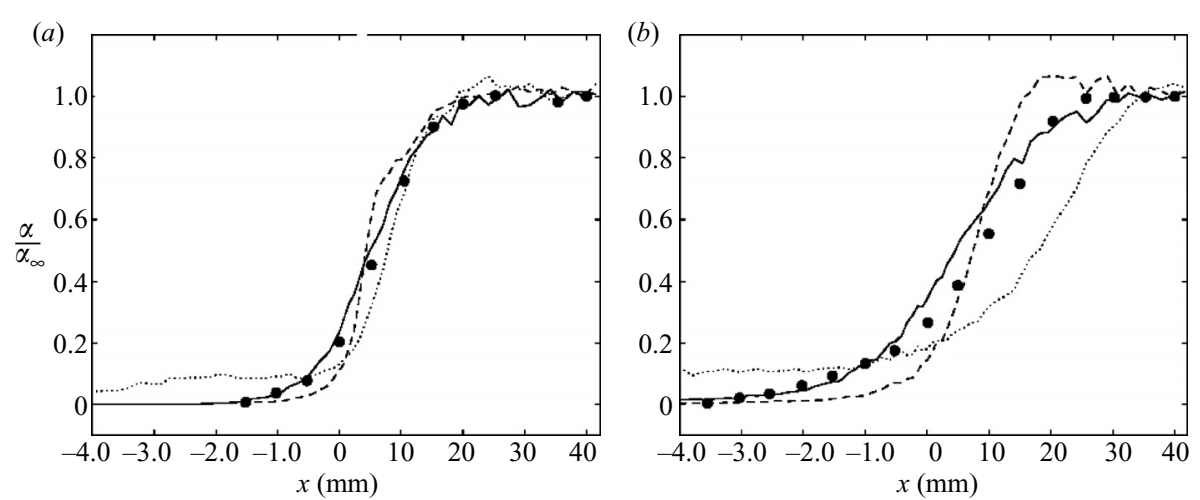

FIGURE 10. Normalized mean liquid to air concentration, $\alpha / \alpha_{\infty}:(a)$ profiles at $z=102 \mathrm{~mm}$, (b) at $z=254 \mathrm{~mm}$. Experiment $(\bullet)$; computed with no SGS dispersion model (dashed line); computed with SGS dispersion model with $\tau_{t}$ given by (3.8) (solid line); computed with SGS dispersion model with $\tau_{t}=\Delta /\left|\boldsymbol{v}_{p}\right|$ (dotted line).

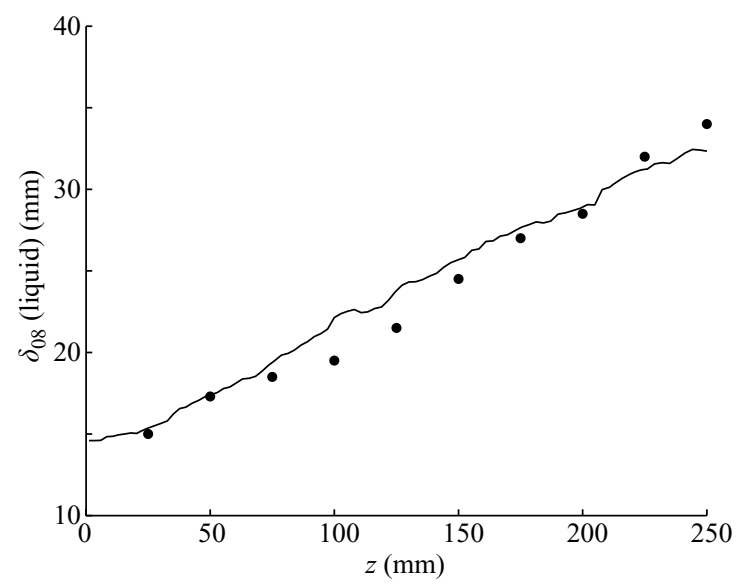

FIGURE 11. Downstream spreading of liquid concentration: $\delta_{08 \text { (liquid), }}$ experiment $(\bullet)$; computed (solid line).

phase. The agreement between the computed and measured results is satisfactory over the entire measurement region, though the computed mean spreading results display a somewhat more linear behaviour than the experimental points, consistent with the linear spreading rate of the gas-phase mixing layer. Further details of droplet transport and cross-stream 'diffusion' can be observed from the joint PDFs of the particle crossstream position and diameter shown in figures 12 and 13. These compare the predicted and measured joint PDF, $P(x, D \mid z)$ at two downstream positions $(z=110 \mathrm{~mm}$ and $250 \mathrm{~mm}$ in the outer portion of the mixing layer. The qualitative shapes of the predicted PDFs resemble very closely the experimental ones. The lack of smoothness of the predicted functions can be attributed to an insufficiently long sampling period; in particular, the number of particles present decreases effectively to zero as the 'edge' of the mixing layer is approached and this requires an increasingly long sampling time to determine droplet statistics and PDFs. Around 150000 stochastic particles were used to represent the spray PDF, $\bar{P}_{s p r}$ at each location. In the experimental work, considerable effort was also devoted to determining the relative differences in the average transport of droplets appertaining to different diameter classes. It was 

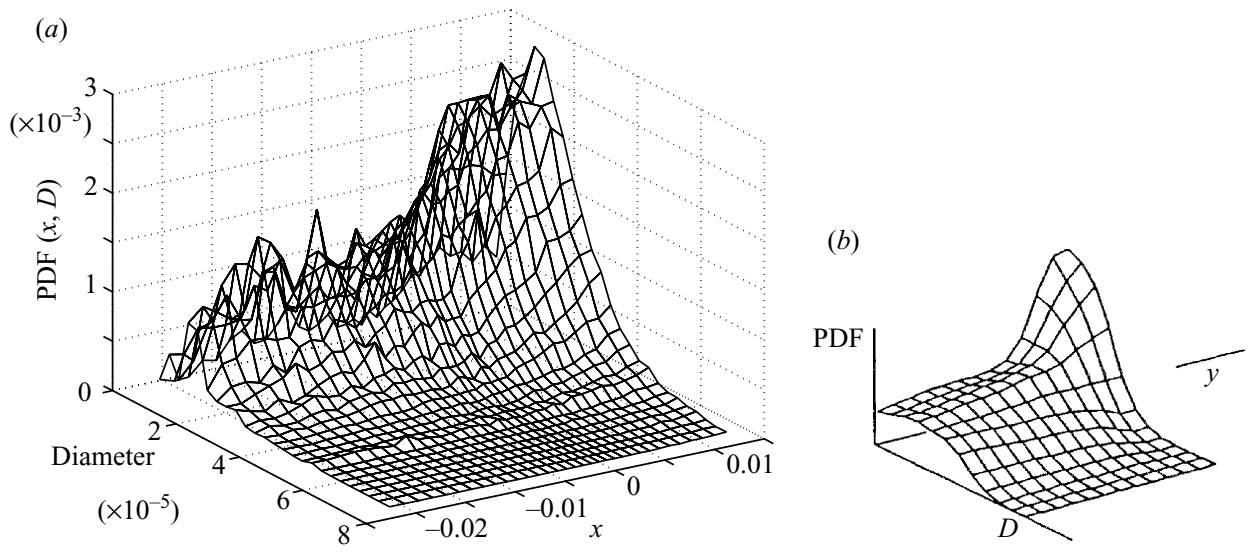

Figure 12. $\operatorname{PDF}(D, x \mid z=100 \mathrm{~mm}):(a)$ computed, $(b)$ obtained in the experiment.
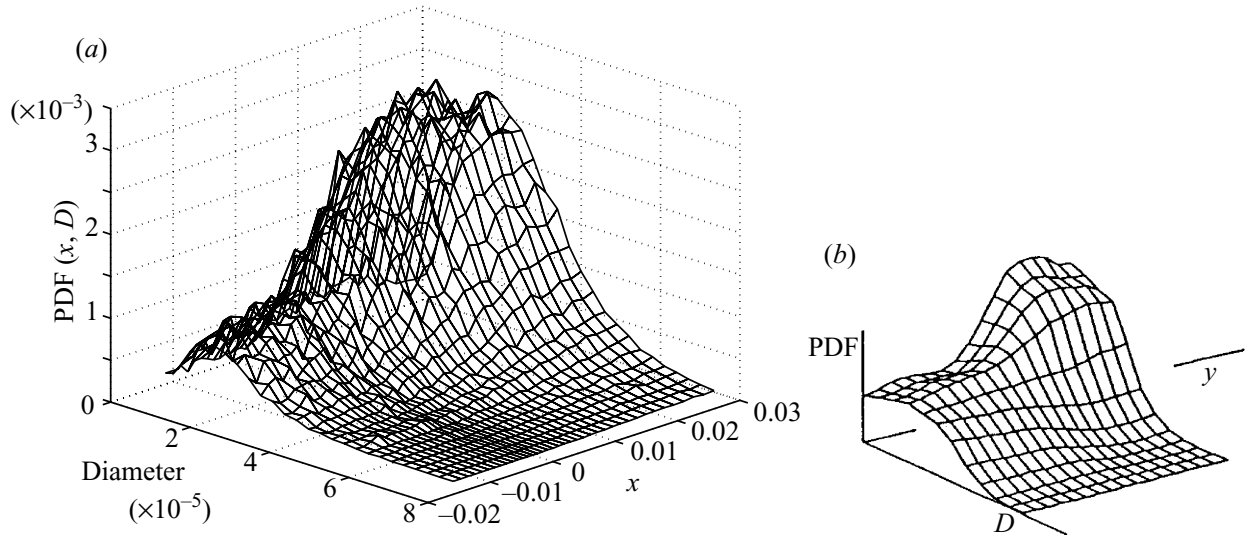

Figure 13. $\operatorname{PDF}(D, x \mid z=254 \mathrm{~mm}):(a)$ computed, $(b)$ obtained in the experiment.

observed that the smaller the particle, the more likely it was to be found in the outer region of the mixing layer, and various results were presented in support of this. The quantities measured included (i) profiles of Sauter mean diameter, SMD, at three different downstream stations, $z^{*}=50,180$ and $250 \mathrm{~mm}$ and (ii) the cross-stream behaviour of the normalized PDF for two different droplet classes, $13 \mu \mathrm{m}$ and $55 \mu \mathrm{m}$ diameter, at the more downstream station: $z^{*}=250 \mathrm{~mm}$.

In order to reproduce those findings, maps of the conditional PDFs, $P\left(D \mid x, z^{*}\right)$ were first computed for $z^{*}=50,180$ and $250 \mathrm{~mm}$ and for a range of $x$-positions; figure 14 shows the result for the downstream location $z^{*}=250 \mathrm{~mm}$. The computations indicate that the ratio of the droplet response time and the Kolmogorov time scale, $\tau_{p} / \tau_{K}$ lies within the range 0.1 to 10 with the value for the majority of droplets being around unity. Consistent with this and as can be readily observed from figure 14, the highest probability arises for small particle diameters towards the outer low-speed edge of the mixing layer, i.e. at negative $x$ ).

It is possible to compute the $\operatorname{SMD}\left(x, z^{*}\right)$ directly from the $\operatorname{PDF}, P\left(D \mid x, z^{*}\right)$. However, in order to reduce the statistical 'noise', as can be seen in figure 14, especially in the outer region, the $\operatorname{PDF}, P\left(D \mid \bar{x}, z^{*}\right)$ from which the $\operatorname{SMD}\left(\bar{x}, z^{*}\right)$ is computed, has been obtained by averaging the one-dimensional PDFs over a small range of $x$ around 


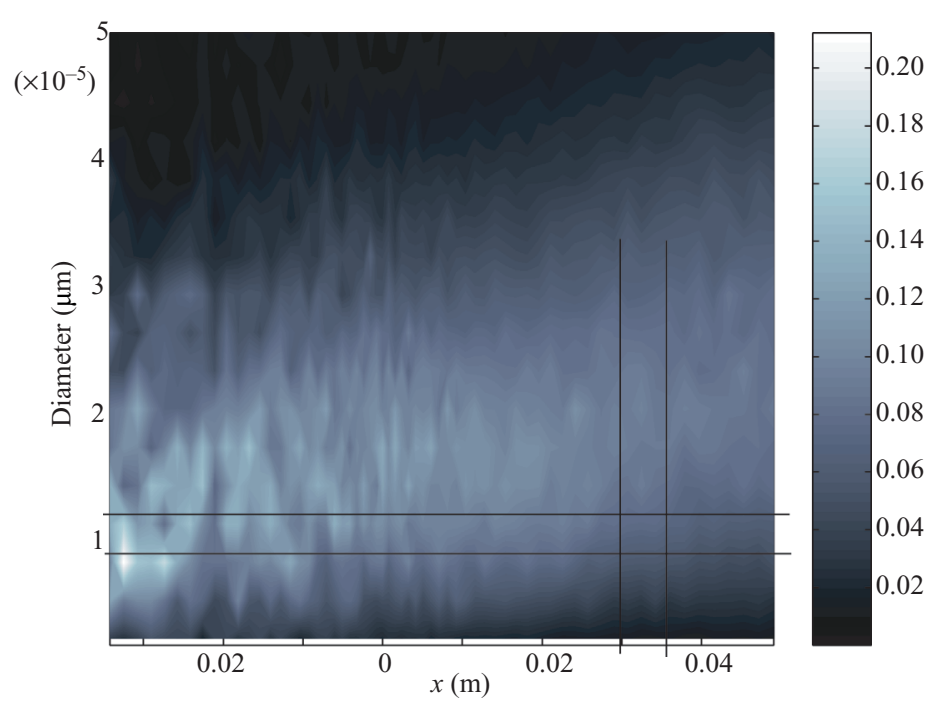

Figure 14. $\operatorname{PDF}(D \mid x, z=254 \mathrm{~mm})$.

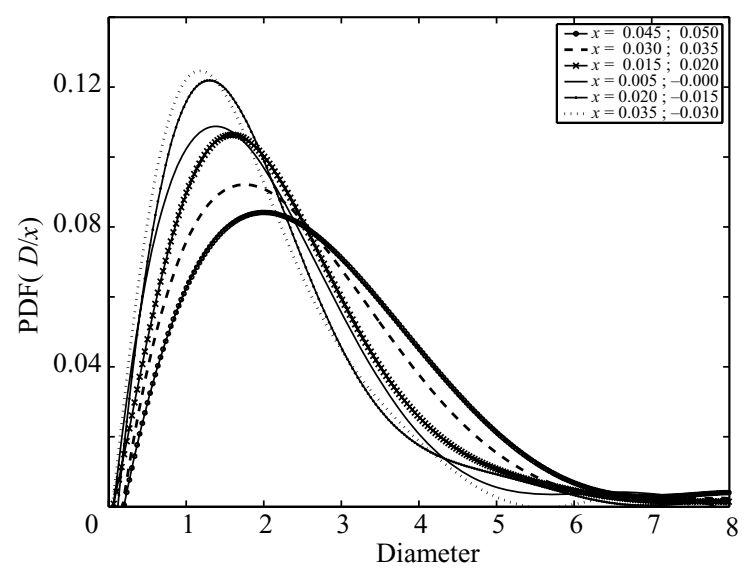

FIGURE 15. Shape extracted from the previous figure for $\operatorname{PDF}(D \mid x, z=254 \mathrm{~mm})$ : each curve is the representative curve for the middle $x$ point of the interval reported in the legend.

$\bar{x}$ and then smoothing the result by interpolating with a fourth-order polynomial. Averaging and interpolation is carried out over an interval of $5 \mathrm{~mm}$, indicated by the two horizontal lines shown in figure 14. Figure 15 shows the particle diameter PDFs, at $z^{*}=250 \mathrm{~mm}$, obtained with this procedure. The particle diameter PDFs deviate appreciably from the analytical shape prescribed at the inflow. The cross-stream profiles of $\operatorname{SMD}\left(x, z^{*}\right)$ computed from the smoothed $\mathrm{PDF}, P\left(D \mid x, z^{*}\right)$ are compared with the measured profiles in figure 16. The agreement between experiment and predictions is good. This, together with the predicted functional shapes of figure 15, suggests that the characteristics of the mixing-layer-droplet dispersion are consistent with experimental observation. The lighter the particles, the more probable it is that they will interact with the turbulent structures of the gas phase in such a way that they migrate towards the external edge of the mixing layer. The relative velocity between the smallest droplets and the continuous phase is likely to be small, with the consequence that their trajectories will be determined by the large-scale vortical 

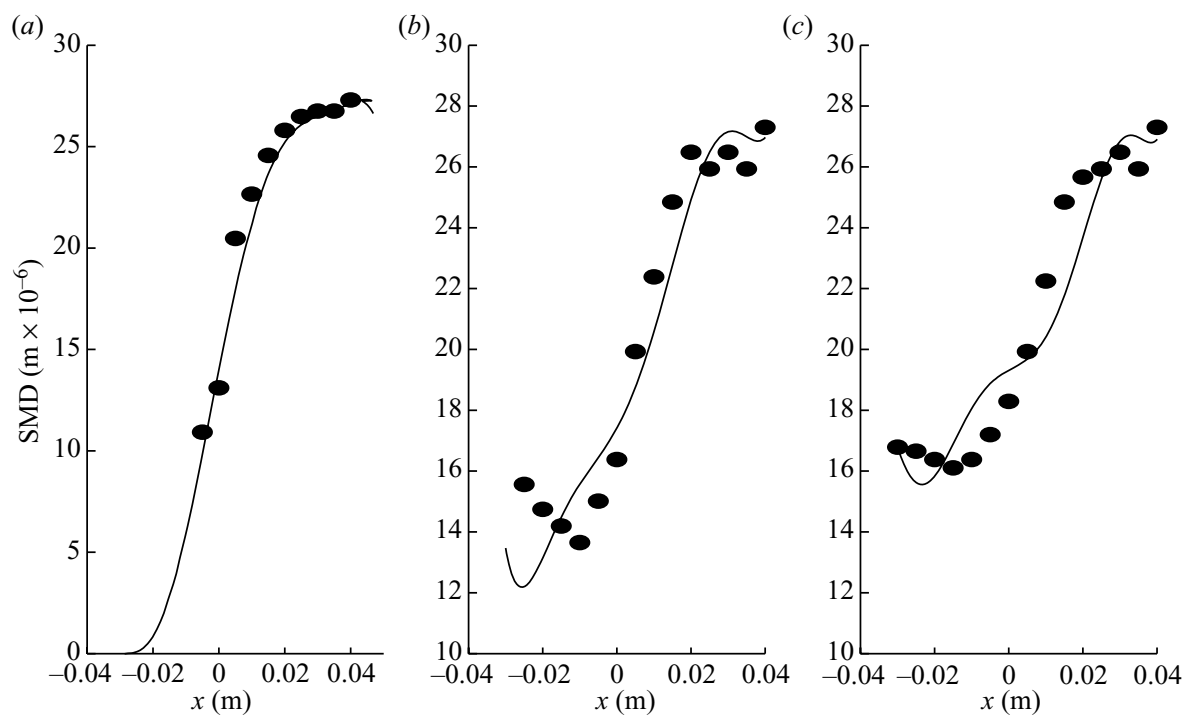

FIGURE 16. Cross-stream distribution of the Sauter mean diameter at three different downstream locations: (a) $z=51 \mathrm{~mm},(b) z=178 \mathrm{~mm},(c) z=254 \mathrm{~mm}$. Computed (solid line); experiment $(\bullet)$.

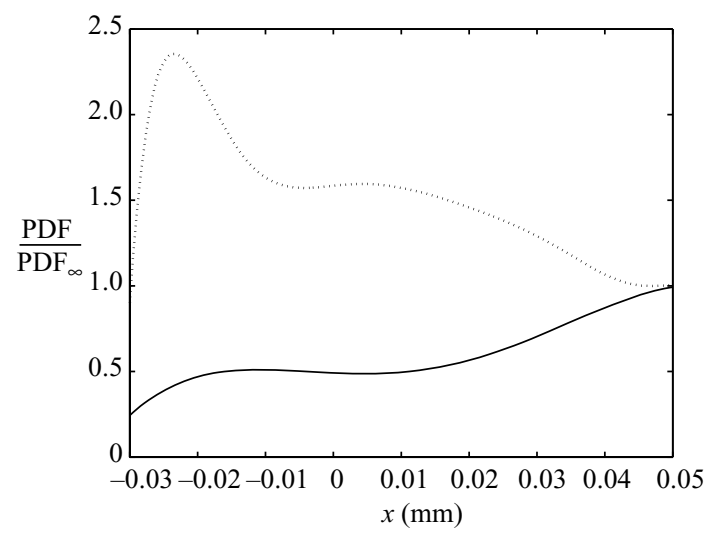

FIgURE 17. Normalized $\operatorname{PDF}\left(x \mid z=254 \mathrm{~mm} ; D=D^{*}\right)$ for two droplet classes: $D^{*}=13 \mu \mathrm{m}$ (dotted line); $D^{*}=55 \mu \mathrm{m}$ (solid line).

structures of the mixing layer, i.e. the small-diameter droplets 'follow' the flow. The influence of the structures is such that small droplets are transported preferentially towards the low-speed side of the mixing layer so that in such a region the particles are characterized by a smaller diameter.

In further support of these observations, the predicted behaviour of the conditional PDF $P\left(x \mid D, z^{*}\right)$ is examined. The procedure adopted to compute these conditional PDFs is similar to the averaging and interpolation used for the PDF shown in figure 14. The resulting PDFs, $P\left(x \mid D, z^{*}\right)$ are plotted in figure 17 for $\bar{D}=13 \mu \mathrm{m}$ and $\bar{D}=55 \mu \mathrm{m}$. The difference in the behaviour of the small and the large droplets at $z^{*}=250 \mathrm{~mm}$ is clearly evident. In particular, the maximum conditional probability that occurs for $\bar{D}=13 \mu \mathrm{m}$ is almost certainly due to the already mentioned clustering effects arising from large-scale vortical turbulence structures discussed above. The 


$\begin{array}{cccc}\text { Computation } & \text { Particles } & \text { Stochastic dispersion active } & \alpha \text { at inflow } \\ \text { I } & \text { No } & - & - \\ \text { II } & \text { Yes } & \text { No } & 10^{-5 \dagger} \\ \text { III } & \text { Yes } & \text { Yes, } C_{0}=1 & 10^{-5} \\ \text { IV } & \text { Yes } & \text { No } & 5 \times 10^{-4} \\ \text { V } & \text { Yes } & \text { Yes, } C_{0}=1 & 5 \times 10^{-4}\end{array}$

$\dagger$ Experimental value

TABLE 1. Characteristics of the computations with sampling. $\alpha=$ [liquid volume/gas volume]

$\begin{array}{cccc}\text { Probe number } & x(\mathrm{~m}) & y(\mathrm{~m}) & z(\mathrm{~m}) \\ 1 & -0.0250 & 0.0500 & 0.0250 \\ 2 & -0.0500 & 0.0500 & 0.1780 \\ 3 & -0.0200 & 0.0500 & 0.1500 \\ 4 & 0.0200 & 0.0500 & 0.1500 \\ 5 & -0.0600 & 0.0500 & 0.2540 \\ 6 & 0 & 0.0500 & 0.3000 \\ 7 & -0.0250 & 0.0500 & 0.3000 \\ 8 & 0.0250 & 0.0500 & 0.3000 \\ 9 & -0.0500 & 0.0500 & 0.3000\end{array}$

TABLE 2. Coordinates and numbering of the probing volumes.

dynamics of smaller droplets are closely linked to those of the gas flow and, as has been observed, they concentrate preferentially at the edges of the large-scale vortical structures of the gas phase turbulence. In the present case, the 'edge' of the mixing layer occurs on average at around $x=-20 \mathrm{~mm}$ at the downstream location $z=250 \mathrm{~mm}$, e.g. figure 4 .

\section{Two-way coupling}

\subsection{Preliminaries}

In order to shed further light on the processes underlying the interactions between the particle and flow fields, it is advantageous to examine the spectral energy content of particle-laden and particle-free flows. The aim here is to elucidate further the influence of the large-scale vortical structures of the gas phase on droplet dispersion and 'mixing' and particle segregation phenomena and to investigate the correlation between the particle and gas flows. With this in mind, time series of the flow and particle fields have been sampled during simulations conducted for the conditions in table 1. Samples have been collected within particular LES cells defined as probing volumes. To enhance the effects of particle concentration, two simulations have been carried out with a particle concentration at the inflow fifty times higher than that of the experiment.

The locations of the probing volumes are reported in table 2. Before the sampling began, a simulation without particles was completed to the point at which the effects of the initial conditions had practically disappeared. This condition was then used as the starting condition for each of the different computations of table 1 . For particle-laden cases, particles were injected into the domain at the inflow at this starting condition. An examination of particle entrainment at different probing locations and particle mass loadings provides a means of determining the influence 

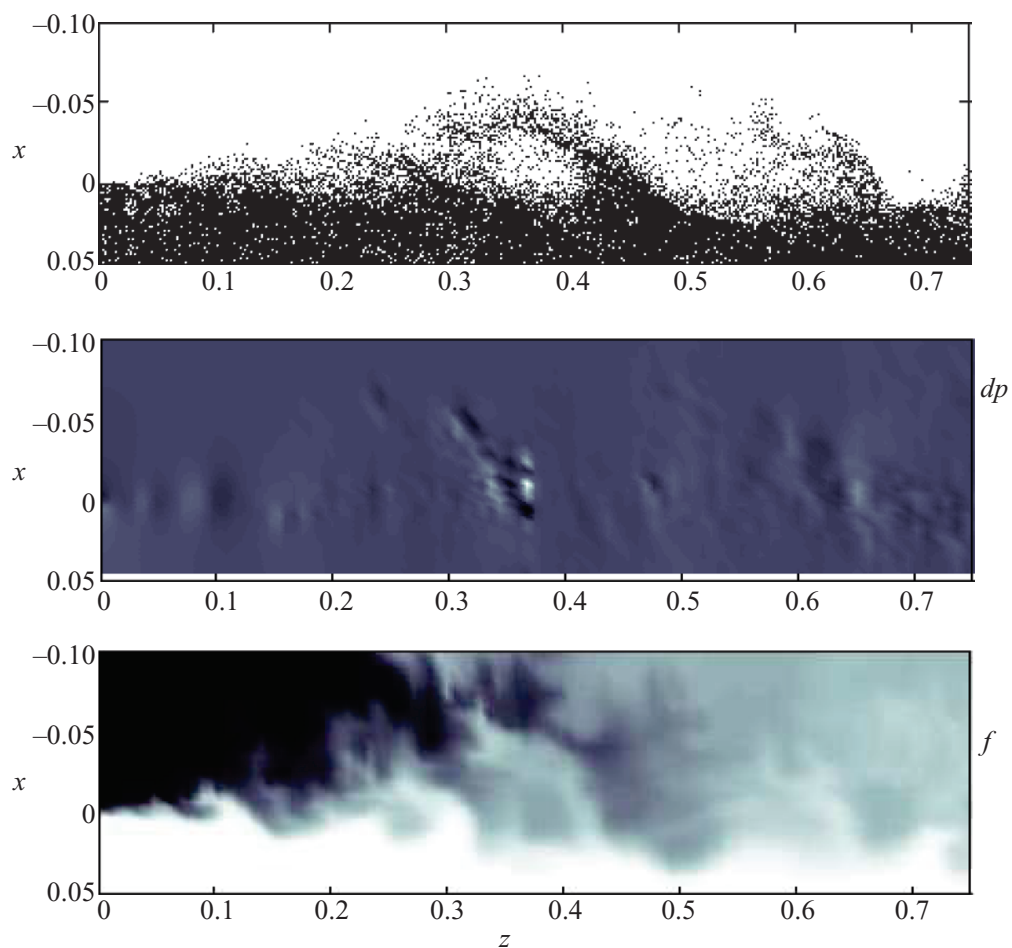

FIGURE 18. Contour maps of the particle field, the pressure and a passive scalar transported by the droplet-laden stream.

of the stochastic dispersion model and the typology of departure from that of the particle-free flow, simulation I. The gradual departure of the various simulations from that of the particle-free flow is illustrated in figure 19 where the time series, determined by probes 3 and 4, of the streamwise velocity are shown whereas, in figure 20, the corresponding time series of the dispersed phase concentration are reported. A straightforward examination of these raw signals reveals that: (i) the time series sampled within the faster moving stream are characterized by a higher frequency content; (ii) the amplitude of the 'dominant' velocity fluctuations increases when particles are transported; (iii) stochastic dispersion is extremely influential in providing higher liquid concentrations in the outer zone; and (iv) the time series of liquid concentration with the stochastic model displays an intermittent character consistent with the dispersion model properties. A qualitative picture of particle behaviour is provided by the snapshot shown in figure 18 from which it is clear that when particles are transported they tend to organize and segregate in preferential locations such that the overall instantaneous shape resembles that resulting from a passive scalar added to the fast-moving droplet-laden inflow stream. To examine these aspects in more detail, Fourier and wavelet analyses are conducted.

\subsection{Fourier analysis}

The Fourier power spectral densities of the $x$ and $z$ components of velocity for the particle-free flow exhibit a marked cross-stream $(x)$ dependence of the more energetic frequencies (figure 21). For probes lying within the faster-moving side of the mixing layer, the signals are characterized by high-frequency fluctuations whereas in the slow-moving side - the outer probing position - lower-frequency modes are excited. 

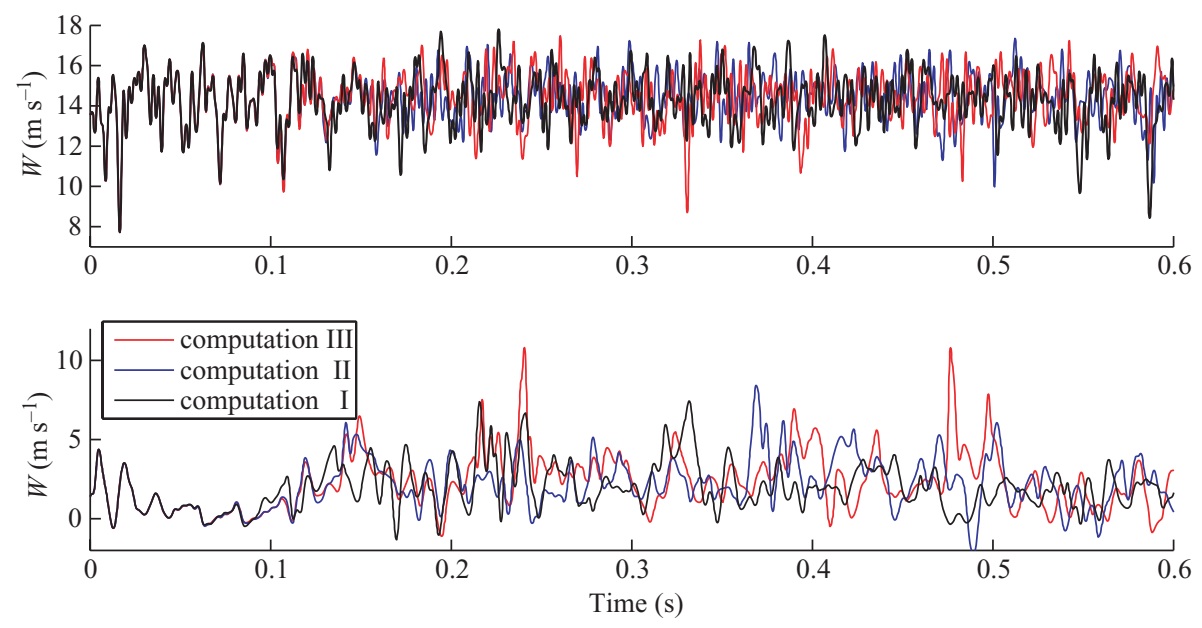

FIGURE 19. Time series of the $z$-component velocity, $w$ for probes 3 and 4.
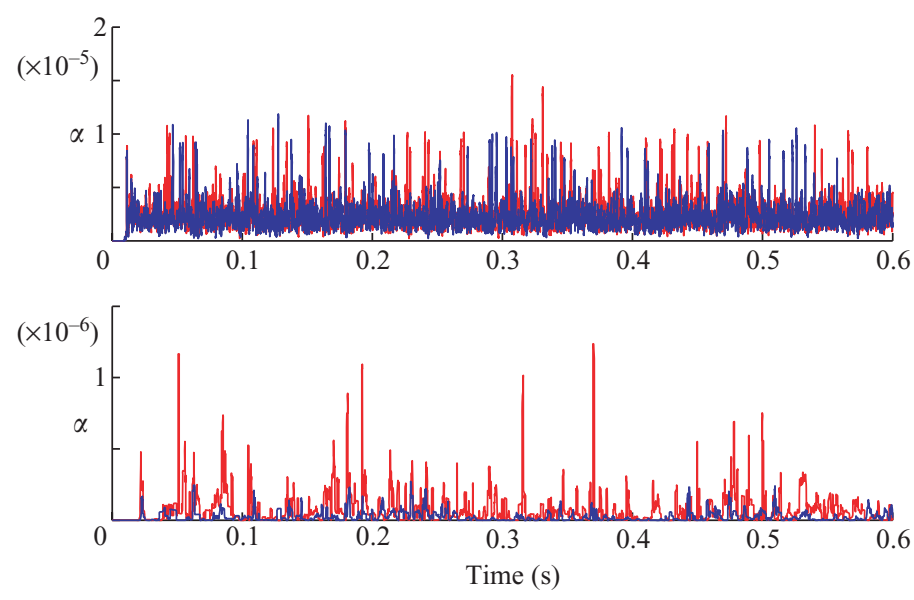

FIGURE 20. Time series of the concentration of the dispersed phase, $\alpha$ for probes 3 and 4 .

Probe 8 exhibits dominant frequencies in the range $80-200 \mathrm{~Hz}$; for probe 6 , the energy is localized in the range $60-70 \mathrm{~Hz} ; 40-50 \mathrm{~Hz}$ is the dominant range for probe 7 ; and very low $(8-10 \mathrm{~Hz})$ high-energy-containing frequencies are displayed for probe 9 . The fact that markedly dominating frequencies can be observed in the spectrum of single components but are in some way smoothed out in the spectra of $|\boldsymbol{v}|$ and $|\boldsymbol{\omega}|$ has been reported also by Lazaro \& Lasheras (1989) and can be attributed to three-dimensional features of the flow.

A comparison of the gas-phase spectra for the cases of particle-free and particleladen flows, with different particle concentrations, reveals two major distinctive features regarding the large-scale energy-containing modes, $f<100 \mathrm{~Hz}$ ). First, some of the energetic modes present in the particle-free flow have an energy content that is enhanced by the presence of particles, whereas the rest of the low-frequency components are generally damped. This aspect can be observed from the power spectral density of the $y$-component of vorticity obtained from probe 6 shown in figure 22 , where the higher energy gain of the dominant vorticity frequencies is evident. 

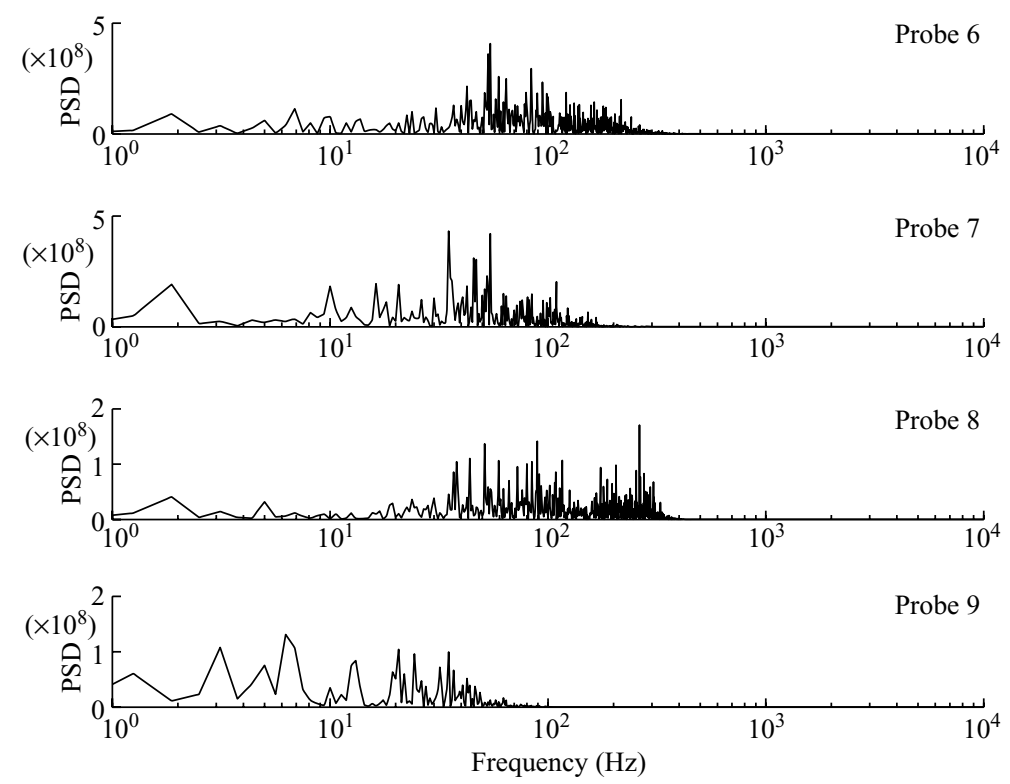

FIGURE 21. Particle-free flow: power spectral densities of the $x$ and $z$ velocity components at various positions across the mixing layer.

In particular, the greater the particle concentration, the higher the energy gain of the dominating vorticity frequencies. Secondly, at certain locations, the dominant frequencies of the non-laden case are damped while at the same time new energetic frequencies appear for the particle-laden case (figure 23). It is tempting to speculate that the presence of the particles acts on the flow by reducing and damping the energy of local perturbations whilst enhancing the energy of the large-scale characteristic structures of the mixing layer. Additional evidence for this is provided in figure 24, where spectra of the $y$-component vorticity is shown for probes 7 and 8 . The particlefree case is characterized by high-energy fluctuations at high frequencies near the boundary. It is unclear whether this behaviour is attributable to high-frequency 'noise' that may have resulted as a consequence of numerical aspects - all non-dissipative discretization schemes are susceptible to this - or to a physical mechanism arising in the near-wall region. However, when particles are present, the energy content of these high frequencies is damped remarkably. The results from probes 7 and 8 show that a few selected frequencies gain energy whilst the energy associated to most of the spectra is damped. The frequencies gaining energy can be thought of as being those characteristic of the large-scale turbulence structures of the mixing layer; the frequency values are noticeably close to the values at which the longitudinal vortices are expected to shed.

The linear power spectra of the time series of liquid concentration, plotted in figure 25 for computations II and III and probes 6-9, show that high-energycontaining frequencies are also found in the dynamics of $\alpha$ and the range of frequencies excited is similar to that appearing in the vorticity spectra. For computation III, where stochastic dispersion is enabled, the high-frequency range appears more likely to be excited. For the simulations with no SGS dispersion, the liquid concentration fluctuations often have enhanced very low frequencies, see for example probe 9. In general, the liquid concentration seems to fluctuate preferentially with frequencies 

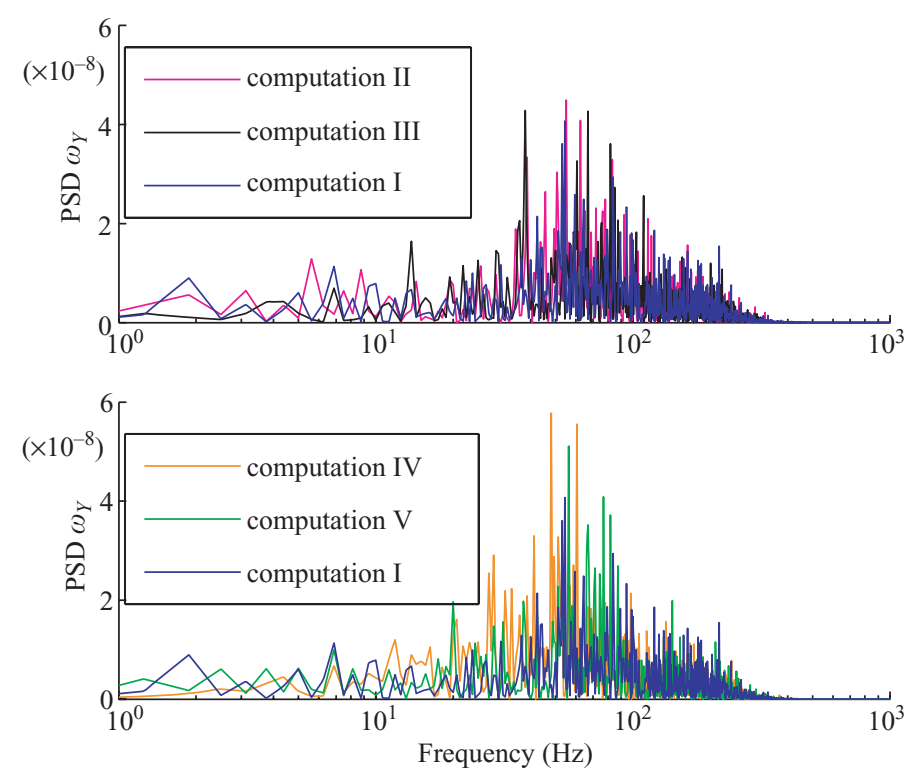

FIGURE 22. Power spectral densities of the $y$-component vorticity obtained from probe 6 .

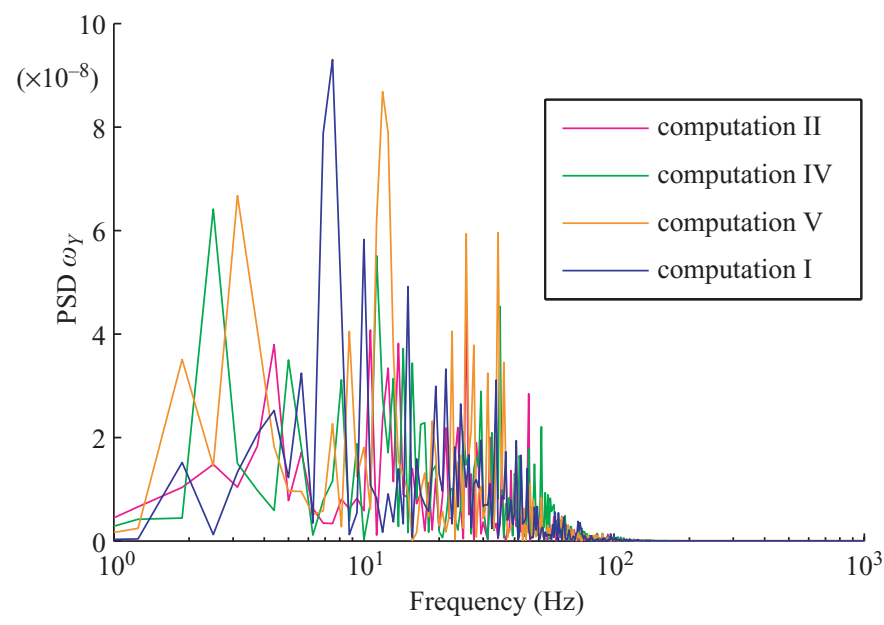

FIGURE 23. Power spectral densities of the $y$-component vorticity obtained from probe 9 .

which are of the same order or somewhat lower than the energetic modes found in the gas phase; such phenomena may be linked to inertial effects characterizing the particle phase. Finally the power spectral densities of streamwise gas-phase velocity and particle concentration are plotted on semi-logarithmic axes for probes 6-9 in figures 26 and 27. At very high frequencies, the spectra exhibit either a linear form, probes 6 and 7, or a wide band of excited modes, probe 9. This is a consequence of sampling at every time step with the result that the signals are over sampled during the computations, the time step is chosen so as to maintain the maximum Courant number (based on the local filtered velocity) at less than of order 0.3. This energy band is obtained only for particle-laden simulations in situations where particle velocity fluctuations, transferred directly to the gas-flow field by the two-way coupling, 

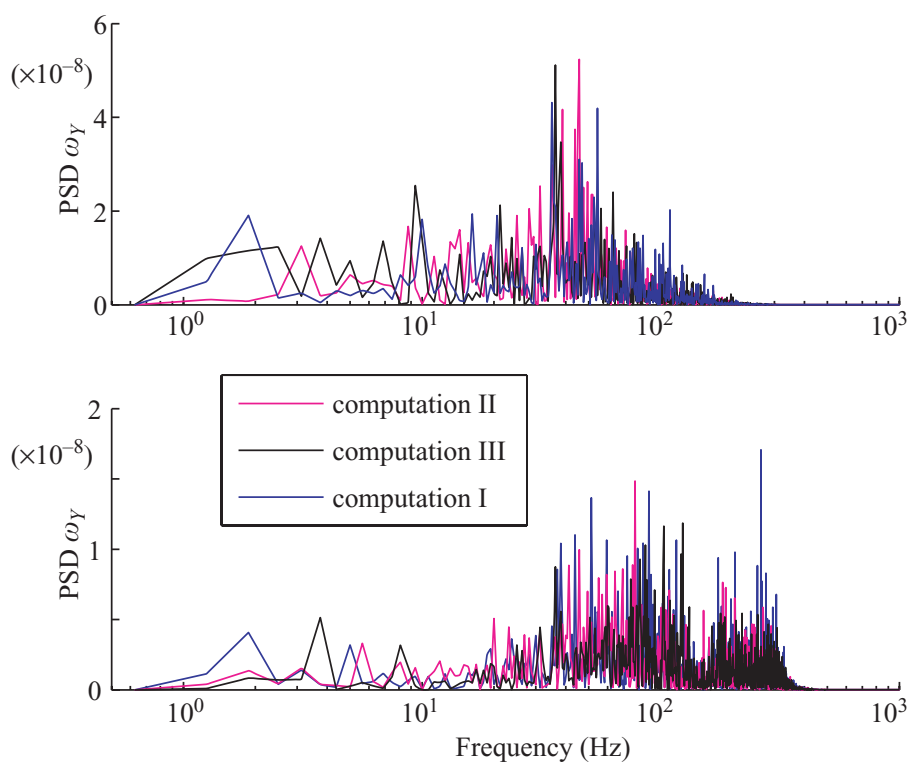

Figure 24. Power spectral densities of the $y$-component vorticity obtained from (a) probe 7 and $(b)$ probe 8 .

are capable of inducing fluctuations at the probing location. Such fluctuations are of a period which is the same order as the integration time step, thus falling in the over-sampled modal region. Notwithstanding this, the instantaneous coupling of the stochastic forces (particle dispersion) acting on the continuous and dispersed phases may result in a high-frequency energy source for the continuous phase the physical nature of which may be questionable. However, it is fundamental to note that the power spectral densities associated to these fluctuations are five to ten orders of magnitude smaller than the power spectral densities of the large-scale energetic modes. It therefore appears that ad hoc modification of the SGS dispersion model is not necessary. If required, then either the coupling between the two phases could be implemented over an averaged set of time steps, thus smoothing out instantaneous random forcing fluctuations or by a modification to the sub-filter residual stress model. The existence of an inertial $(-5 / 3)$ range can be identified in most of the plots though the extent to which it applies is variable, dependent on the probe position. This is consistent with the observation that the large-scale structures are characterized by frequency ranges which depend on the location; if the spatial resolution is similar, then the part of the inertial range resolved must be variable. When particles are present, the major changes observed are: (i) a general damping of the large scales which can also be observed in log scale for certain probes, for example probe 9 (the damping is not uniform, a few frequencies are enhanced but this is clearer on linear coordinates); (ii) a small increase in the energy at high frequencies, in the range 100 to $500 \mathrm{~Hz}$, regardless of the inclusion or otherwise of stochastic sub-filter particle acceleration.

\subsection{Wavelet analysis}

Given the evidence of preferential particle concentrations that have been attributed to the influence of the large-eddy structures in the gas phase and given that the flow and the particle concentration fields show similar ranges of excited modes, it is of 
(a)
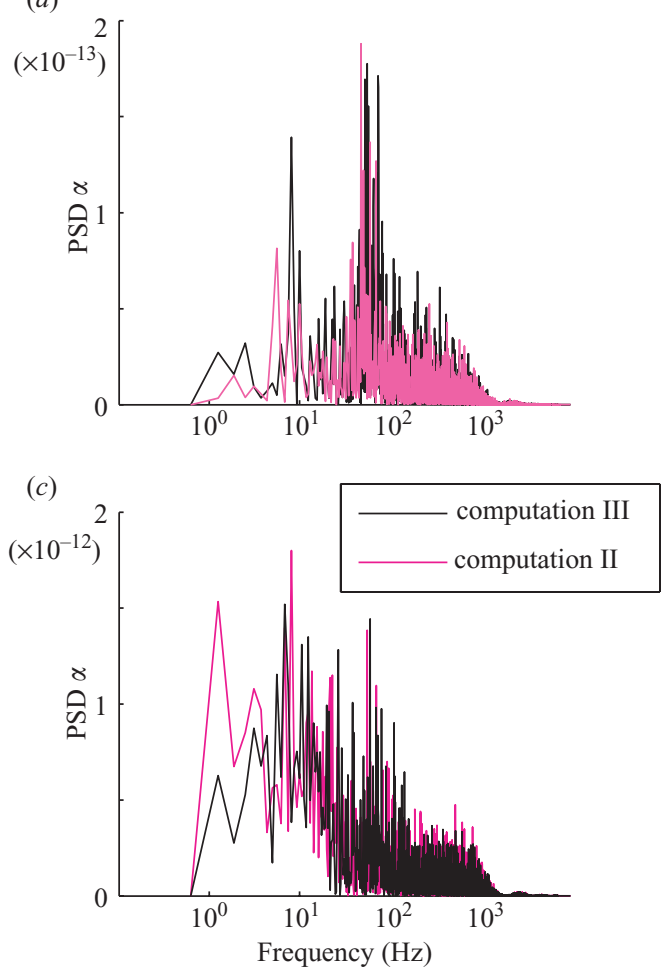

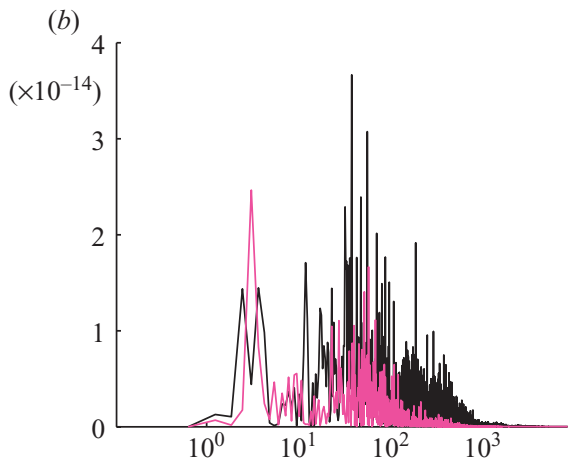

(d)

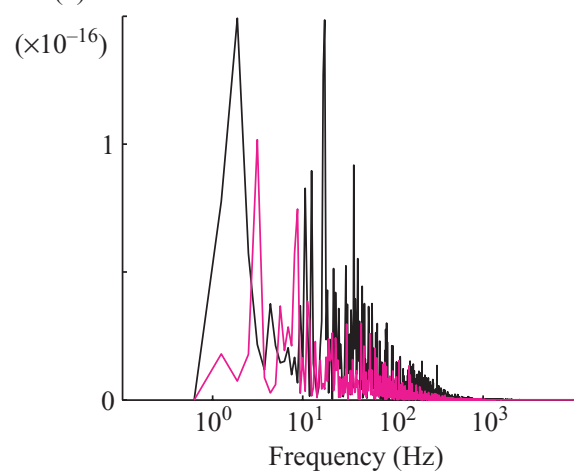

FIgURE 25. Power spectral density of the dispersed phase concentration. (a) Probe 6 , (b) probe $7,(c)$ probe $8,(d)$ probe 9.

interest to investigate the localized time-dependent relation between these two fields. The wavelet framework constitutes an ideal means of doing this as it is eminently non-local, i.e. it enables the analysis of correlations conditioned upon the observation of particular events, and uses basis functions - mother wavelets of eddy-like shape which makes the method eminently suited to capturing and describing large-scale turbulence structures. The Fourier power spectral densities provide only an overall picture of which frequencies are present and how energetic they are during the complete time history.

The wavelet analysis is conducted using a 'Morlet' mother wavelet with $f_{0}=6$. (For a self-contained introduction to statistical signal processing using wavelets and how these can be applied to analyse local events, coherent structures and temporal correlations, see Kaiser 1994; Torrence \& Compo 1998). In what follows, $W_{u}(s, \tau)$ and $W_{u}(f, \tau)$ indicate the wavelet transform of the $u$ time series as a function of scale and time $(s, \tau)$ and frequency and time $(f, \tau)$, respectively, for Morlet $f=f_{0} /(2 \pi s)$. The cross-scalogram for the signals $u$ and $v$ is $W_{u v}(s, \tau)=W_{u}(s, \tau) W_{v}^{*}(s, \tau)$ with * indicating the complex conjugate. This is customarily decomposed according to:

$$
W_{u v}(s, \tau)=C o W_{u v}(s, \tau)-i Q u a d W_{u v}(s, \tau) .
$$

With the relatively benign hypothesis of regularity of the time series, the following holds for the real part of the cross-scalogram:

$$
\int_{-\infty}^{\infty} f(t) g(t) \mathrm{d} t=\frac{1}{C_{\psi}} \int_{0}^{\infty} \int_{-\infty}^{\infty} \operatorname{Co} W_{u v}(s, \tau) \mathrm{d} s \mathrm{~d} \tau,
$$



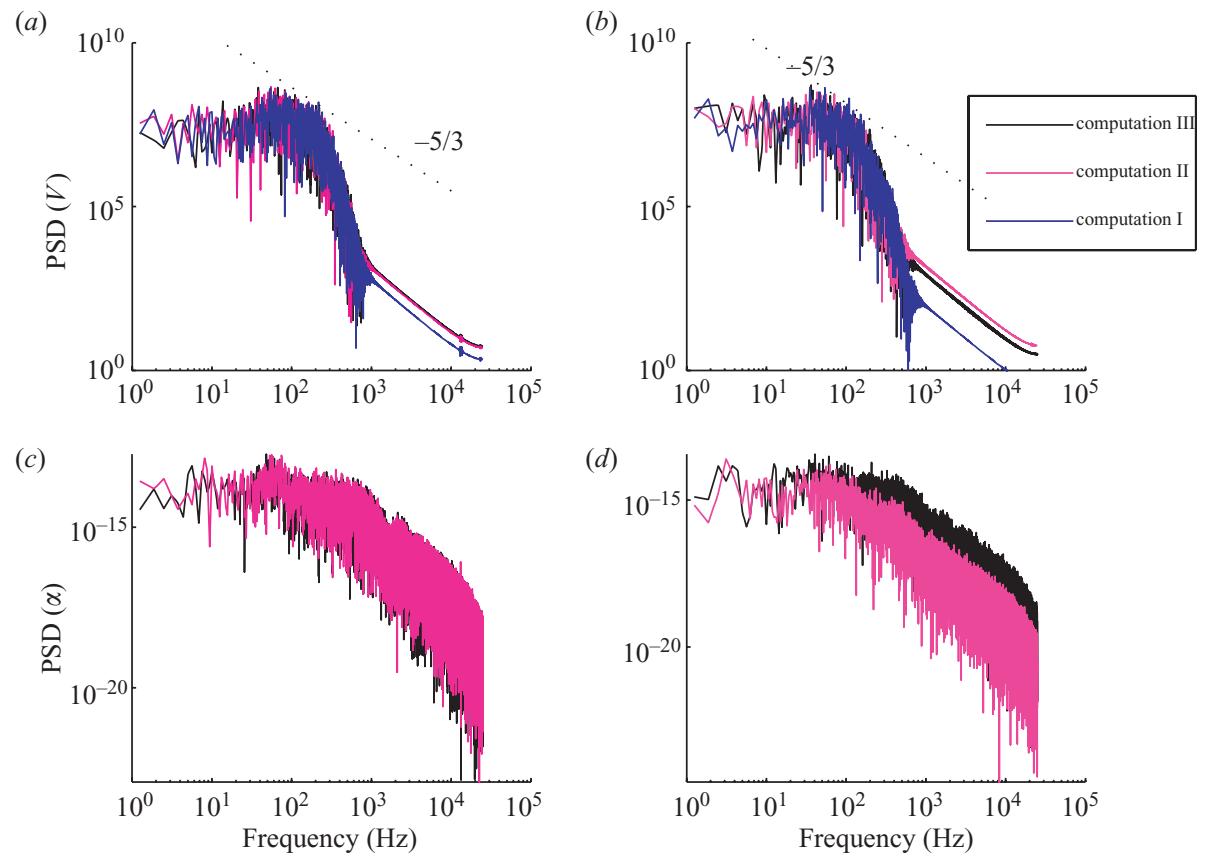

FIGURE 26. Power spectral density of the streamwise gas-phase velocity and dispersed phase concentration; $(a, c)$ probe 6 and $(b, d)$ probe 7 .

where $C_{\psi}$ is the admissibility constant of the wavelet. The above highlights the importance of the $\mathrm{Co}$-cross-scalogram in the investigation of the correlation of $u$ and $v$ and a wavelet local correlation coefficient (WLCC) can be defined:

$$
\operatorname{WLCC}_{u v}(s, \tau)=\frac{C o W_{u v}(s, \tau)}{\left|W_{u}(s, \tau) \| W_{v}(s, \tau)\right|} .
$$

It is straightforward to prove that WLCC $\in[-1,1]$.

Attention is now focused on the time series sampled by probe 6 where the Fourier analysis suggested that most of the features regarding mixing-layer structures exhibit appreciable energy. In figure 28 , the maps of the $y$-component of vorticity, $\left|W_{\left|\omega_{Y}\right|}(f, \tau)\right|^{2}$ and the liquid volume fraction, $\left|W_{\alpha}(f, \tau)\right|^{2}$ are reported for computation II. The timescale evolution of the vorticity component is dominated, for given time instants, almost exclusively by a single structure (a band of excited frequencies) having a width of $20-30 \mathrm{~Hz}$. The energetic band of frequencies is not stationary in time; it oscillates in frequency space. In addition also the intensity - indicated by the colour in the map of the phenomena changes. The overall picture provided of the turbulence structures is more detailed than that suggested by ordinary spectral analysis. There is no structure having a wide band of excited modes as Fourier analysis might suggest. Instead, there exists a relatively narrow band of excited modes whose frequency localization and intensity change in time. The corresponding Fourier spectrum may be seen as related to the wavelet map through some type of time average (see Kaiser 1994). Regarding the liquid concentration, much less coherence can be observed in $\left|W_{\alpha}(f, \tau)\right|^{2}$ for computation II. However, coherent structures appear sporadically and they are often characterized by the same scale range as that of the vorticity component.

The effects produced by an augmented liquid concentration, computation IV, are shown in figure 29. Figures 28 and 29 suggest that an augmented particle concentration 

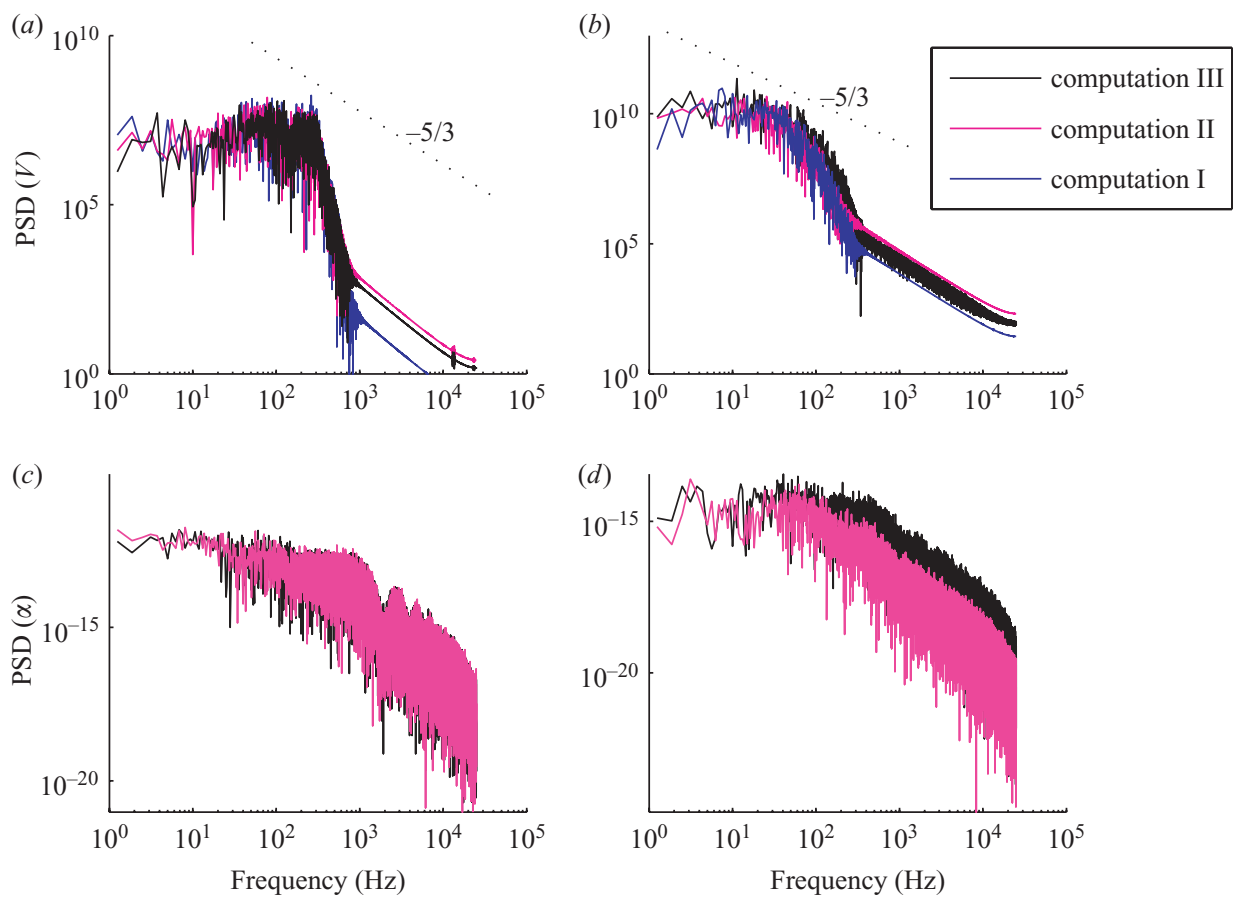

FIGURE 27. Power spectral density of the streamwise gas-phase velocity and dispersed phase concentration; $(a, c)$ probe 8 and $(b, d)$ probe 9 .

tends to enhance the formation of distinctive blobs in the map which represent largescale vortical structures. In scale-time space the mixing-layer structures are definitely more marked and separated in the case of an augmented particle concentration, i.e. they are not blended in an almost continuous pattern as for figure 28. This fact, together with Fourier analysis, which showed how few low-frequency modes gain energy when particles are present, supports the idea that the presence of particles tends to strengthen the most important vortical structures while damping the formation of less intense and subsidiary eddies, at least for the range of particle diameters used in this experiment. The particle diameters vary from $1 \mu \mathrm{m}$ to $200 \mu \mathrm{m}$, the majority being concentrated within the interval $[10,50] \mu \mathrm{m}$ in order to offer a comparison with the classification reported in Ferrante \& Elghobashi (2003), the corresponding particle response times, $\tau_{p}^{(S)}$ resulting from Stokes law lie in the range $\left[10^{-5}, 10^{-3}\right]$ with the majority of particles having $\tau_{p}^{(S)} \approx 10^{-4}$. The Kolmogorov time scale at the centre of the domain can be estimated to be of the order $\tau_{K} \approx 10^{-4.5} \mathrm{~s}$ and this gives a time-scale ratio $\tau_{p} / \tau_{K} \approx \in[0.1,10]$ and for the majority of the particles $\tau_{p} / \tau_{K} \approx 1$. The augmented particle concentration has a large effect on the vorticity field, which, becoming more 'organized', has the effect of enhancing the preferential concentrations of the particle field. The concentration field is highly coupled to the vorticity field as is evident from the more intense and identifiable structures in the fields of $\left|W_{\alpha}(f, \tau)\right|^{2}$ in figure 29 compared with figure 28.

In order to extract information which is hidden in the $|\omega|$ spectra owing to threedimensional effects, wavelet analysis is applied to examine the correlation between the fields of vorticity and particle concentration. For vorticity, attention is focused on $\omega_{y}$ and the results are plotted in figure 30. It is well known (Kaiser 1994) that 

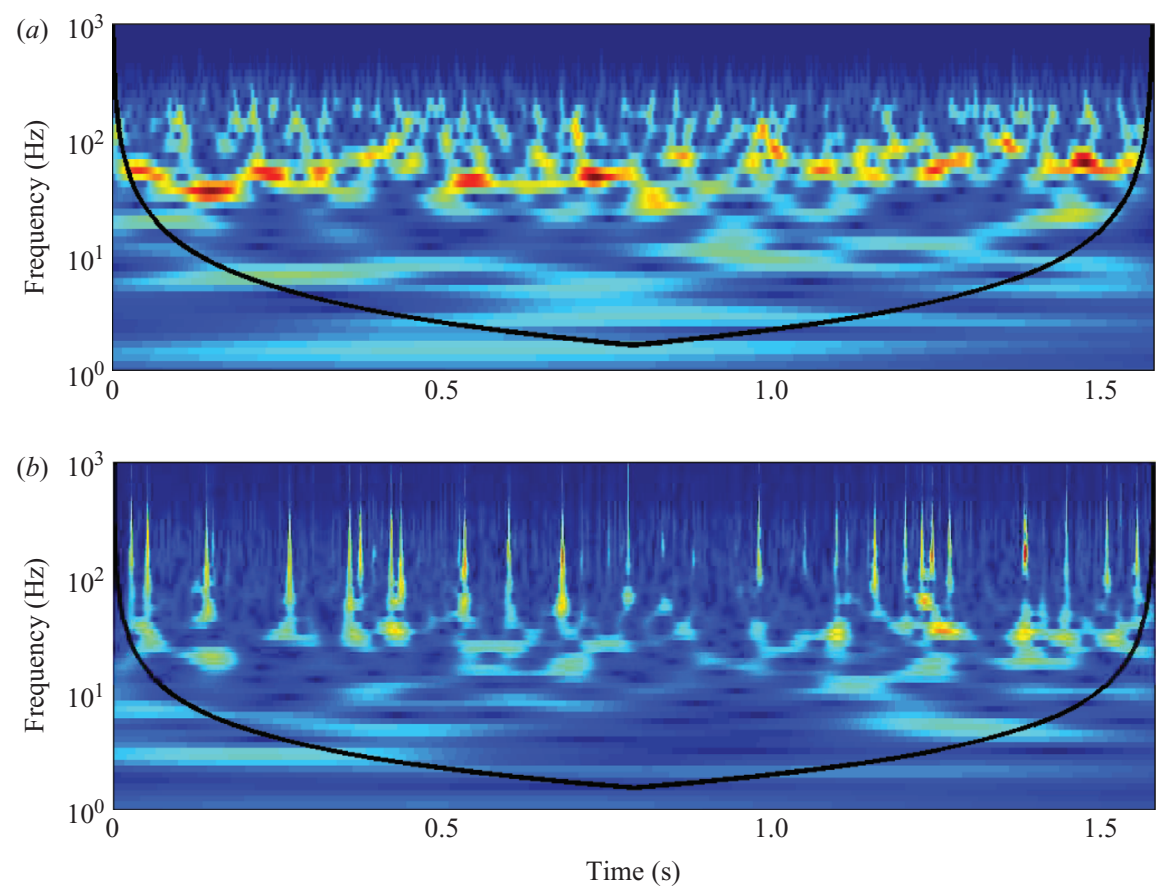

FIGURE 28. Wavelet transform of the time series of vorticity and liquid concentration of computation II. (a) Wavelet map of the energy in the vorticity time series, $\left|W_{\omega}(f, \tau)\right|^{2}$; (b) wavelet map for the energy in the liquid concentration $\left|W_{\alpha}(f, \tau)\right|^{2}$.
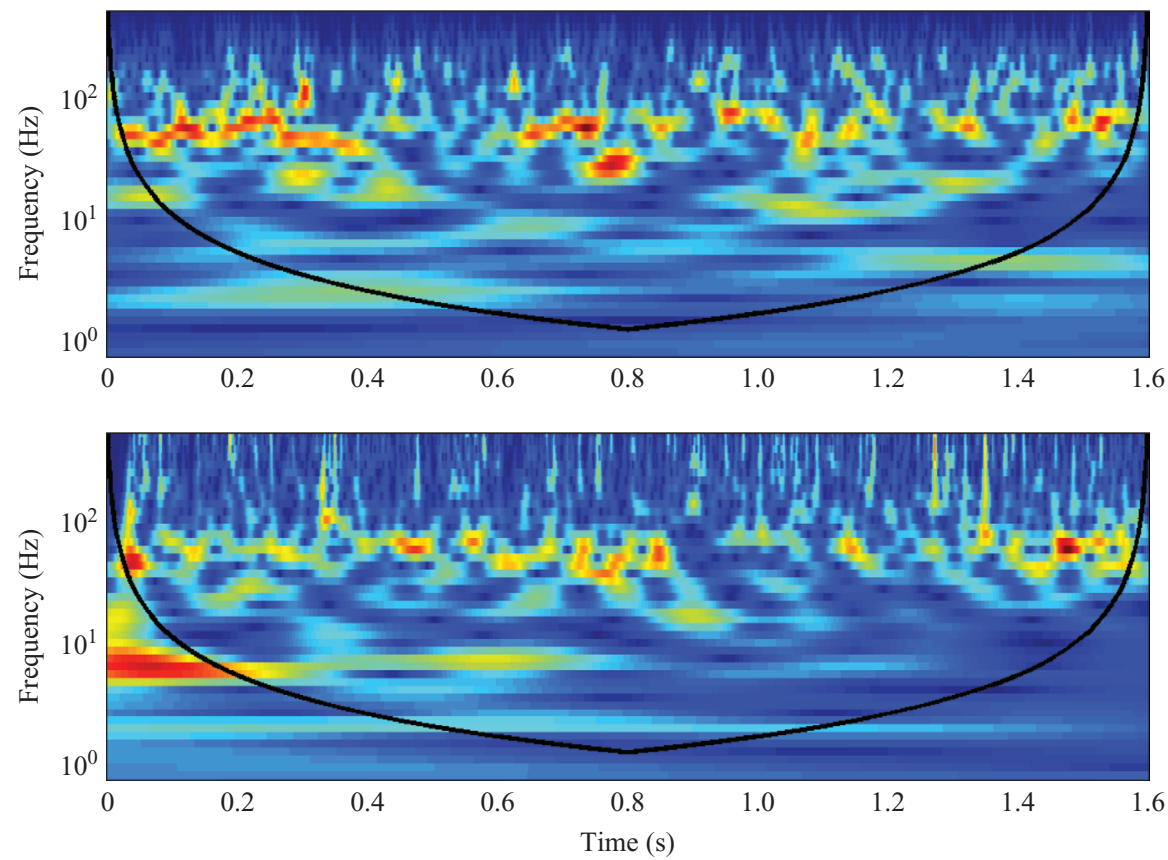

Figure 29. As figure 28, but for computation IV. 

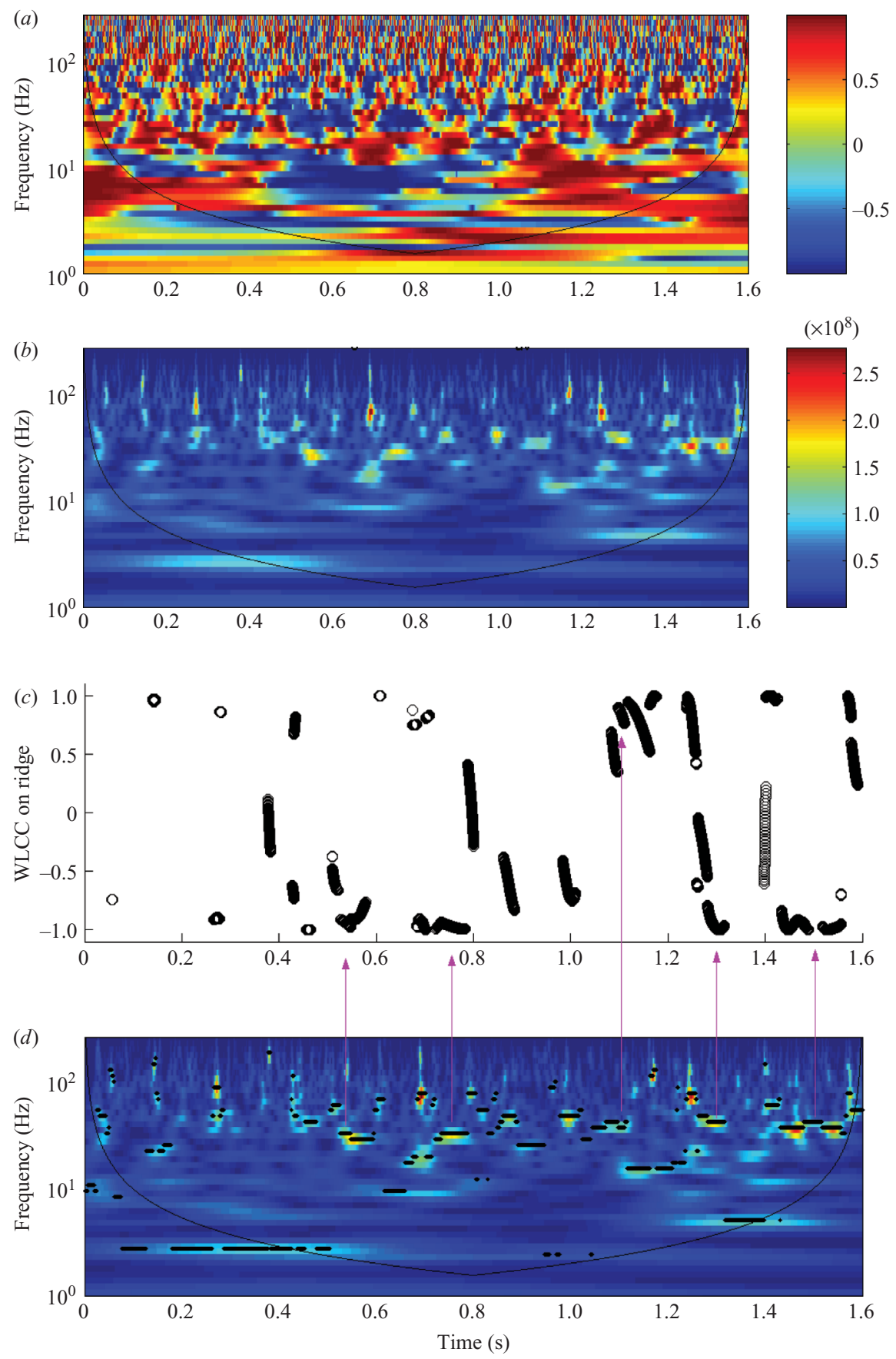

FIGURE 30. Wavelet analysis and wavelet cross-spectral analysis for the time series of vorticity and liquid concentration of computation II. (a) Wavelet map for the vorticity time series, $W_{\omega}(f, \tau),(b)$ for the liquid concentration $W_{\alpha}(f, \tau)$. $(c)$ Wavelet cross-correlation coefficient of vorticity and liquid concentration $\mathrm{WLCC}_{\omega-\alpha} .(d)$ Wavelet cross-spectrum of vorticity and liquid concentration $W_{\omega-\alpha}$. WLCC value along the ridge of the wavelet map of $W_{\omega-\alpha}$; ridge of $W_{\omega-\alpha}$. 
careless observation of the map of the wavelet cross-correlation coefficient of two signals, $u$ and $v, \operatorname{WLCC}_{u v}(s, \tau)$ may point misleadingly at correlations in the timescale domain which are not connected to important energy-containing structures. The remedy for this is to observe the local correlation under a particular template: the energy contained in the wavelet cross-scalogram, $\left|W_{u v}(s, \tau)\right|$. The cross-scalogram highlights the zones where the mutual wavelet transform is more intense. Hence when the correlation, WLCC, in these zones, is examined, the correlation is known to indicate important mutually related physical oscillations. Because it is impracticable to carry out a study involving direct comparison of all the two aforementioned maps, for example $\left|W_{\alpha\left|\omega_{y}\right|}(f, \tau)\right|$ and $\mathrm{WLCC}_{\alpha\left|\omega_{y}\right|}(f, \tau)$ (figure $30 a, b$ ), a procedure is required in order to identify the regions where the correlation should be examined. The procedure followed for extracting physically relevant correlations is, first, to identify a zone where observations are meaningful in the cross-scalogram $\left|W_{\alpha\left|\omega_{y}\right|}(f, \tau)\right|$ and, secondly, to restrict the $W L C C_{\alpha\left|\omega_{y}\right|}(f, \tau)$ to the identified zone. The region where mutual influences are important is defined as the crest or 'ridge' of the wavelet crossscalogram. There appear to be many different ways of defining a 'ridge' available in the literature on wavelet cross-spectral analysis. In the present context, a simple definition is adopted: the ridge is taken to be the locus of the local maxima of $\left|W_{\alpha\left|\omega_{y}\right|}(f, \tau)\right|$ for any given $\tau$.

The procedure described above is applied to the study of local cross-correlation between the fields of $\alpha$ and $\left|\omega_{y}\right|$ shown in figure 30. Arrows have been drawn to indicate the important zones of the ridge which are linked to the mutual interactions between $\alpha$ and $\left|\omega_{y}\right|$, arising from the vortices previously discussed. The WLCC evaluated along the ridge for computations II and IV takes only occasionally positive values and, in the few cases where this happens tends, in a notable manner, toward a perfect positive correlation $(+1)$. The zones where the correlation is -1 can be seen to correspond to time instants where frequencies of the order of 50 to $80 \mathrm{~Hz}$ dominate the cross-scalogram. This suggests that, at the particular range of frequencies typically associated with large-scale mixing structures, vorticity and particles tend to be exclusive. Such an observation confirms in time-frequency space what is visible in the instantaneous fields, i.e. that vortex cores expel particles that are consequently preferentially found at the edges of consecutive eddies.

A similar localized cross-correlation analysis is carried out (figure 31) of the fields of particle concentration and dissipation rate for case II. Here, it is evident that the WLCC of particle concentration and dissipation, when evaluated along the ridge of the scalogram, tends to be remarkably close to +1 , i.e. the dissipation rate is nearly perfectly correlated to the particle field. When particle concentration is above (below) the mean particle concentration at a particular location, the dissipation rate is likely to be above (below) the mean rate of dissipation. However, in spite of this observed strong correlation, it appears likely that there may be no direct causal relationship between dissipation rate and particle correlation. Rather, the present analysis suggests that these two fields, though correlated, are related to each other via an intermediate physical entity, the enstrophy - a measure of the intensity of the vorticity field. With $\leftrightarrow$ being read as 'influences and it is influenced by', a more logical connection to be made seems to be as follows:

$$
\text { Particle field } \leftrightarrow \text { enstrophy (vorticity field) } \leftrightarrow \text { dissipation field. }
$$

The relation between enstrophy and dissipation rate in turbulent flows has been the object of many studies. Amongst others the computational study of Kida \& Ohkitani (1992) and the experiments of Zeff et al. (2003) in a single-phase flow showed how an 

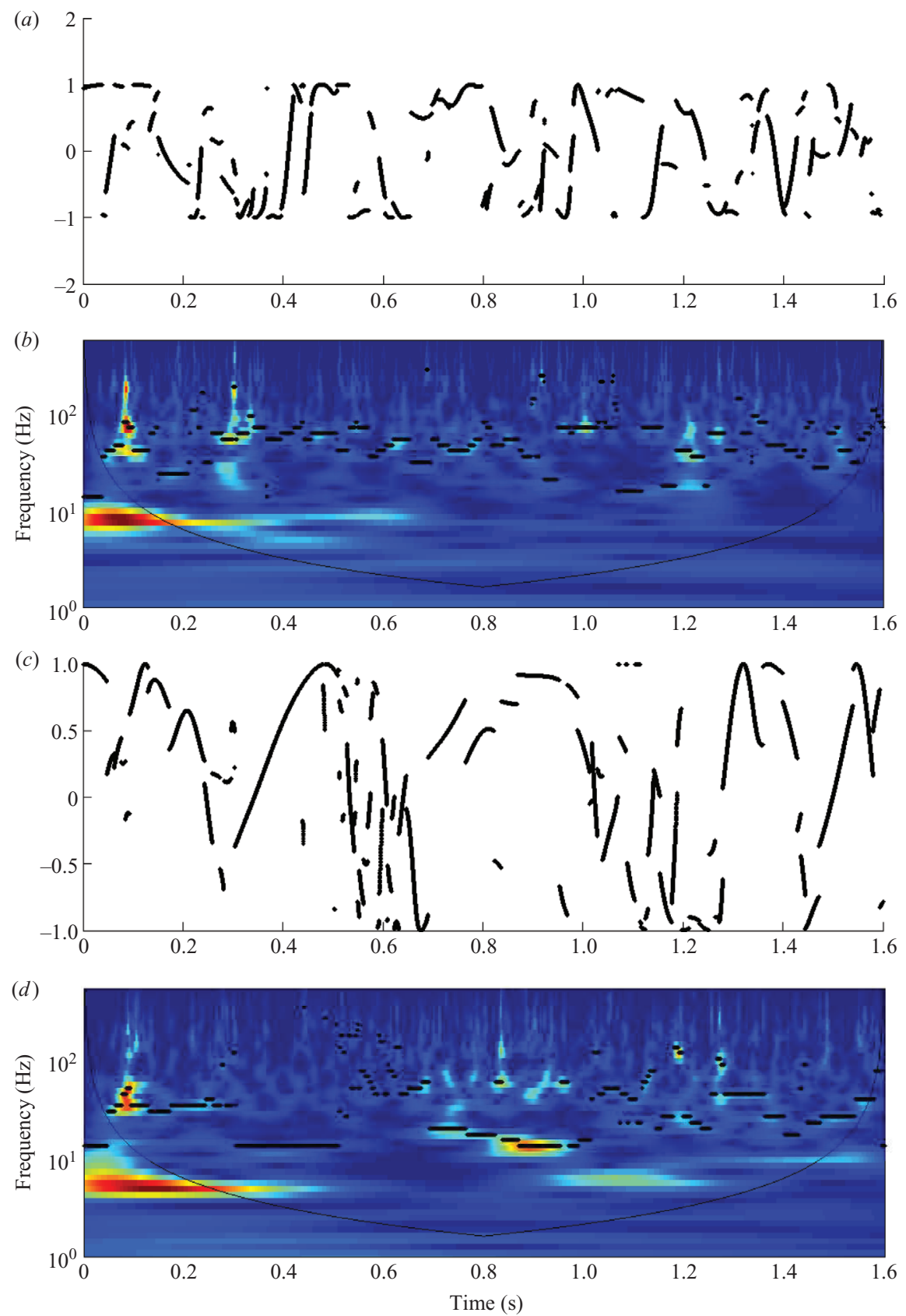

FIGURE 31. Wavelet cross-spectral analysis for the field of dissipation and particle concentration of computations IV and $\mathrm{V}$ at probing location 6. (a) $\mathrm{WLCC}_{\epsilon \alpha}$ along ridge of the cross-scalogram, $(b)$ cross-scalogram $W_{\epsilon \alpha}(f, \tau)$ for computation IV; $(c)$ WLCC $_{\epsilon \alpha}$ and (d) $W_{\epsilon \alpha}(f, \tau)$ for computation $\mathrm{V}$.

increase in enstrophy is accompanied to a decrease in dissipation and vice versa. The same strong connection is observed here, see figure 32, where the cross-spectral wavelet analysis is carried out for the field of vorticity and dissipation rate. Along the ridge of the cross-scalogram, the field of $\left|\omega_{y}\right|$ and dissipation have a WLCC which is almost 

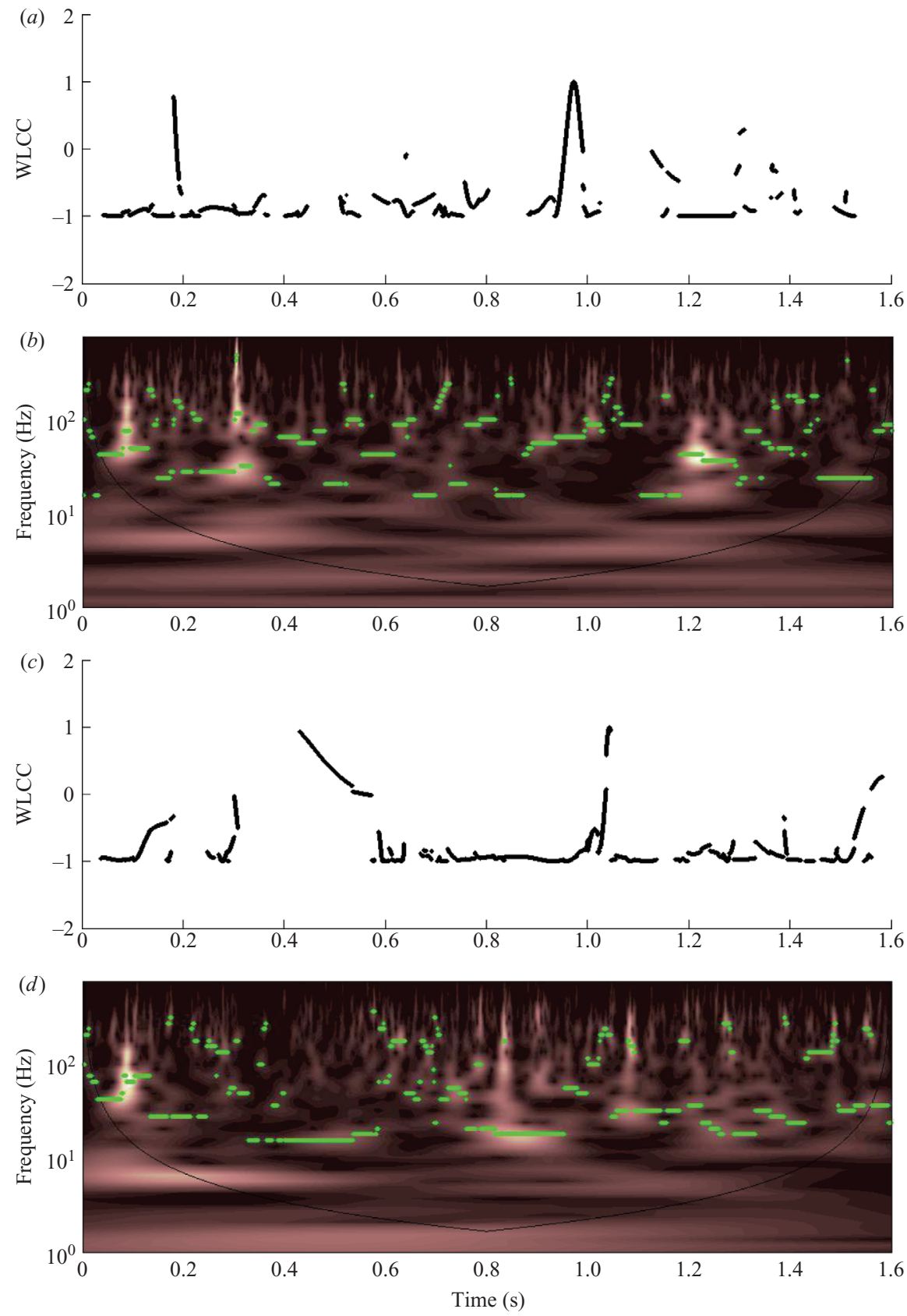

FIGURE 32. Wavelet cross-spectral analysis for the field of dissipation and vorticity of computations IV and $\mathrm{V}$ at probing location 6. (a) $\mathrm{WLCC}_{\left|\omega_{Y}\right| \alpha}$ along ridge of the cross-scalogram, $(b)$ cross-scalogram $W_{\left|\omega_{Y}\right| \alpha}(f, \tau)$ for computation IV; $(c) \mathrm{WLCC}_{\left|\omega_{Y}\right| \alpha}$ and (d) $W_{\left|\omega_{Y}\right| \alpha}(f, \tau)$ for computation $\mathrm{V}$.

everywhere -1 . This finding is in agreement with the view that dissipation tends to be relatively small at the centre of a vortex, where a small amount of deformation is present in comparison to rotational effects, and that it increases with increasing distance from the vortex centre to have a high value at the 'edge' of a vortex where 
there is high strain and small rotation. The fact that dissipation rate and vorticity are so well negatively correlated and that, as previously shown, the particles can provide a means whereby large-scale vorticity structures acquire longevity, supports the idea that the interactions between particle motion and turbulence are best understood in terms of the influence of particles on the enstrophy field, and the consequence that this has on the field of dissipation rate.

\section{Conclusions}

The concept of LES filtered probability density function has been applied to provide a probabilistic description of the dispersed liquid droplet phase in the context of LES of two-phase flows. Models for closing the unknown terms in the transport equation for the joint filtered probability density function of the dispersed phase properties have been proposed. In particular, following a heuristic procedure, it has been show how SGS dispersion effects can be modelled in a stochastic manner; during the present work, attention has been focused on providing a physically accurate representation of the droplet subgrid scale dispersion effects. To achieve this a multi-dimensional isotropic Wiener process based on a particle response time has been proposed. The conditions necessary for extending the formulation to a more general anisotropic model have been outlined in Appendix A.

The capabilities of the approach have been evaluated in detail by application to the particle-laden mixing layer studied experimentally by Lazaro \& Lasheras (1989, $1992 a, b)$. In doing so, it has been shown that SGS dispersion plays a crucial role in determining the spreading and properties of the dispersed phase. The good agreement achieved between the LES and experimental results is dependent on the choice of the particle response time as being representative of subgrid dispersion. The LES results obtained with the formulated model have been analysed in some detail and have been found to be entirely consistent with the behaviour observed experimentally. Practically identical conclusions on droplet behaviour with respect to size and distribution can be drawn from the LES and experimental results. The work thus constitutes a step towards the accurate prediction and optimization of spray flows of practical interest.

The good agreement obtained in the statistical quantities motivated the use of the computational results in an investigation of the particle-turbulence interactions in a shear flow. The spectral modulation of turbulence and flow characteristics owing to particle transport shows how large scales of the flow can gain energy and acquire longevity through the influence of particle motion. This is due to the capability of large-scale vortical structures to segregate particles at their edges, thus increasing their rotational inertia. This feature appears not to have been observed previously in numerical studies as these were carried out in isotropic turbulence where the entire energy spectral content of the flow is damped by the presence of particles. Identification of large-scale structures and the study of localized crosscorrelation coefficients between the fields of particle, enstrophy, and dissipation also provides insight into the particle-turbulence interactions. In particular, the present study suggests that the connection between particle concentration and turbulence dissipation fields can be better understood if, rather than examining their direct correlation, the effects of both fields upon the field of vorticity are investigated.

The authors gratefully acknowledge the financial support provided by the the European Community under the project INTELLECT D. M. (Project AST3-CT2003-502961). 


\section{Appendix A. Anisotropic diffusion tensor}

A random vector is a finite-dimensional column whose entries are random variables, quoting Feller (1968): 'A random vector is to be viewed as a vector with a random length and random direction'. If in a physical vector, the length is the vector invariant, then in a random vector, the invariant can be thought to be its length in probability. In order to define a distance between random variables, the concept of a norm must be introduced; let $X$ be a random variable defined on a certain probability space $(\Omega, \mathscr{F}, P), X: \Omega \rightarrow \mathfrak{R}^{n}$, for positive integers $k \geqslant 1$ and the $k$ th norm can now be defined as:

$$
\|X\| k=\left[E\left(|X|^{k}\right)\right]^{1 / k},
$$

where $|X|$ represents the usual Euclidean norm in $\Re^{n}$ and $E(\ldots)$ the expectation with respect to the probability $P$. The $k$-norm, like any norm, can be used to measure distance; the $k$-distance (or $k$-metric) between real-valued random variables $X$ and $Y$ is defined:

$$
d_{k}(X, Y)=\|X-Y\| k=\left[E\left(|X-Y|^{k}\right)\right]^{1 / k} .
$$

All the moments of $X$ about $a$ are simply the $k$-distances from $X$ to $a$. Two random variables can be said to be identical in probability if $d_{k}(X, Y)=0, \forall k$.

The aim here is to construct a stochastic process representing the unresolved particle acceleration starting from a standard Wiener process in $\mathfrak{R}^{n}$. A natural way of doing so is by supposing that the variance of the process is proportional to the (estimated) subgrid-scale kinetic energy. In this simple way of proceeding, note that: different observations of the flow field using different frames of reference will have the same kinetic energy estimate, independent of the reference frame, and so the stochastic processes should remain unchanged. If a particle travelling through a region where the turbulent fluctuations are strongly anisotropic is considered, then a possible method of accounting for this is to construct a random vector such that the modelled particle acceleration is a function of the anisotropy. However, the resulting stochastic process must satisfy the invariance of random vector lengths: different observers using different frames of reference to obtain the anisotropy of the flow field at a given point must build random vector processes that are the same in probability. Hence:

$$
\|d v\|_{k}=\left[E\left(\left|\boldsymbol{B} d \boldsymbol{W}_{t}\right|\right)^{k}\right]^{1 / k},
$$

where $\boldsymbol{B} \in \mathfrak{R}^{n \times n}$ is the diffusion matrix and $\boldsymbol{W}_{t} \in \mathfrak{R}^{n}$ is the standard $n$-dimensional Wiener process. To simplify, let us consider the case $n=2$, and let us suppose that the modelled diffusion matrix is taken to be anisotropic and diagonal. Let the result arising from two different reference frames be $\boldsymbol{B}_{O 1}=\operatorname{diag}\left[\sigma_{1}, \sigma_{2}\right]$ and $\boldsymbol{B}_{O 2}=\operatorname{diag}\left[\lambda_{1}, \lambda_{2}\right]$, respectively. Let us now suppose that the two different frames of reference differ only by a solid-body rotation through an angle $\theta$. To guarantee the invariance of vector norms, we must ensure that the two diffusion matrices become identical when written in the same reference frame. If $T(\theta)$ is the rotation matrix then:

$$
\boldsymbol{B}_{O 1}=T^{-1}(\theta) \boldsymbol{B}_{O 2} T(\theta)=\left(\begin{array}{ll}
\lambda_{1} \cos ^{2}(\theta)+\lambda_{2} \sin ^{2}(\theta) & \cos (\theta) \sin (\theta)\left(\lambda_{2}-\lambda_{1}\right) \\
\cos (\theta) \sin (\theta)\left(\lambda_{2}-\lambda_{1}\right) & \lambda_{2} \cos ^{2}(\theta)+\lambda_{1} \sin ^{2}(\theta)
\end{array}\right) .
$$

Hence, if the model is to be reference-frame independent, then the diffusion matrix can be a diagonal matrix if and only if it as isotropic. It is thus necessary to preserve the appropriate tensor transformation properties in any modelled diffusion matrix that purports to incorporate the effects of subgrid-scale anisotropy. With this in mind, 
the following simple model can be constructed:

$$
\boldsymbol{B}_{i j}=\frac{\widetilde{u_{i} u_{j}}}{k_{s g s}} \times \sqrt{\frac{C_{0}}{\tau} k_{s g s}}
$$

where $\widetilde{u_{i} u_{j}}$ can be represented by:

$$
\widetilde{u_{i} u_{j}}=\frac{2}{3} k_{s g s} \delta_{i j}-2 v_{s g s} \tilde{S}_{i j}
$$

where $v_{s g s}$ is the SGS eddy viscosity and where $\tilde{S}_{i j}=1 / 2\left(\partial \tilde{u}_{i} / \partial x_{j}+\partial \tilde{u}_{j} / \partial x_{i}\right)$. Finally, an estimate of the SGS kinetic energy can be obtained from:

$$
k_{s g s}=\left(2 \Delta v_{s g s} \tilde{S}_{i j} \tilde{S}_{i j}\right)^{2 / 3} .
$$

\section{Appendix B. Inflow data generation based on digital filters}

In this Appendix, the main features of the work of Klein et al. (2003) for the generation of turbulent inflow data are summarized. The usual approach to the generation of synthetic turbulence inflow data is to generate a velocity signal that has certain statistical properties, which may, for example, be known from experimental data. Such quantities are mean values, the Reynolds stresses, higher-order moments, energy spectra, two-point velocity correlations and various length and time scales. Such a procedure can be split into two parts: first a provisional three-dimensional signal $\mathscr{U}_{i}$ possessing prescribed properties, typically two-point statistics (length scale, energy spectra) is generated for each velocity component; then the required Reynolds stress tensor can be obtained using a transformation method, such as that proposed by Lund, Wu \& Squires (1998). In order to create a two-point correlation, let $r_{m}$ be a series of random data with zero mean and unity variance, the quantity

$$
u_{m}=\sum_{n=-N}^{N} b_{n} r_{m+n}
$$

then defines a convolution or a digital linear non-recursive filter. The quantity $u_{m}$ can be interpreted as being the value of one component of the velocity vector at a discrete point in space or time, corresponding to a single realization. The $b_{n}$ are the filter coefficients and $N$ is connected to the support of the filter. Because $\left\langle r_{m} r_{n}\right\rangle=0$ for $m \neq n$ it follows that:

$$
\frac{\left\langle u_{m} u_{m+k}\right\rangle}{\left\langle u_{m} u_{m}\right\rangle}=\sum_{j=-N+k}^{N} b_{j} b_{j-k} / \sum_{j=-N}^{N} b_{j}^{2},
$$

and this determines the relationship between the filter coefficients and the twopoint correlation function of $u$. A three-dimensional filter can be obtained from the convolution of three one-dimensional filters:

$$
b_{i j k}=b_{i} b_{j} b_{k} \text {. }
$$

Rather than attempt to construct a detailed form for the correlation function, it is presumed that it depends only on the separation distance, $r=|\boldsymbol{r}|$ and a single length scale. The following shape is adopted:

$$
R_{u u}(r, 0,0)=\exp \left(-\frac{\pi}{4} \frac{r^{2}}{L^{2}}\right)
$$


Suppose $\Delta x$ is the grid spacing and $L=n \Delta x$ the desired length scale, then the correlation function can be written in discretized form as:

$$
\frac{\left\langle u_{m} u_{m+k}\right\rangle}{\left\langle u_{m} u_{m}\right\rangle}=R_{u u}(k \Delta x)=\exp \left(-\frac{\pi k^{2}}{4 n^{2}}\right),
$$

where the filter coefficients are given by:

$$
b_{k} \approx \tilde{b}_{k} /\left(\sum_{j=-N}^{N} \tilde{b}_{j}^{2}\right)^{1 / 2}, \quad \tilde{b}_{k}:=\exp \left(-\frac{\pi k^{2}}{2 n^{2}}\right) .
$$

With the filter coefficients previously defined, it is possible to filter a collection of random maps differing for only a row or a column of random numbers. If the random numbers are shifted ad hoc between subsequent filtering operations, it is possible to obtain filtered fields of data in which every field has a spatial correlation and each point of a given filtered field is correlated with the same point of some of the subsequent filtered maps.

\section{REFERENCES}

Ahmed, A. M. \& Elghobashi, S. 2000 On the mechanism of modifying the structure of turbulent homogeneous shear flows by dispersed particles. Phys. Fluids 12, 2906-2930.

Aliseda, A., Cartellier, A., Hainaux, F. \& Lasheras, J. C. 2002 Effect of preferential concentration on the settling velocity of heavy particles in homogeneous isotropic particles. J. Fluid Mech. 468, 77-105.

Bellan, J. 2000 Perspectives on large eddy simulation for sprays: issues and solutions. Atomiz. Sprays 10, 409-425.

BINI, M. 2006 Large eddy simulation of particle and droplet laden flows with stochastic modelling of sub-filter scales. PhD thesis, Imperial College, University of London, London, (UK).

BinI, M. \& JoNES, W. P. 2007 Particle acceleration in turbulent flows: a class of non-linear stochastic models for intermittency and heavy tailed pdfs. Phys. Fluids 19 (3), 035104.

Boivin, M., Simonin, O. \& SQuires, K. D. 1998 Direct numerical simulation of turbulence modulation by particles in isotropic turbulence. J. Fluid Mech. 375, 235-263.

Bosse, T., Kleiser, L. \& Meiburg, E. 2006 Small particles in homogeneous turbulence: settling velocity enhancement by two-way coupling. Phys. Fluids 18, 027102.

Branley, N. 1999 Large eddy simulation of non-premixed turbulent flames. PhD thesis, Imperial College, University of London, London, UK.

Branley, N. \& JONES, W. P. 1999 Large eddy simulation of a turbulent non-premixed swirling flame. In Engineering Turbulence Modelling and Measurements 4 (ed. D. Lawrence \& W. Rodi), pp. 861-870. Elsevier.

Branley, N. \& Jones, W. P. 2001 Large eddy simulation of a turbulent non-premixed. Combust. Flame 127, 1914-1934.

Buyevich, Y. A. 1971 Statistical hydromechanics of disperse systems. Part 1. Physical background and general equations. J. Fluid Mech. 49, 489-507.

BuYEvich, Y. A. 1972a Statistical hydromechanics of disperse systems. Part 2. Solution of the kinetic equation for suspended particles. J. Fluid Mech. 52, 345-355.

Buyevich, Y. A. $1972 b$ Statistical hydromechanics of disperse systems. Part 3. Pseudo-turbulent structure of homogeneous suspensions. J. Fluid Mech. 56, 313-336.

Crowe, C. T. 1982 Numerical models for dilute gas-particle flows. Trans. ASME I: J. Fluids Engng 104, 297-305.

Dopazo, C. 1994 Turbulent Reactive Flows: Recent Developments in PDF Methods, chap. 7, pp. 375-474. Academic.

DruZHININ, A. 2001 The influence of particle inertia on the two-way coupling and modification of isotropic turbulence by micro-particles. Phys. Fluids 13, 3738-3755.

Elghobashi, S. \& Truesdell, G. C. 1993 On the two way interaction between homogeneous turbulence and dispersed solid particles. I: Turbulence modification. Phys. Fluids A 5, 1790-1801. 
Feller, W. 1968 Probability Theory and Applications. Wiley.

Ferrante, A. \& Elghobashi, S. 2003 On the physical mechanism of two way coupling in particleladen isotropic turbulence. Phys. Fluids 15, 315-330.

Fessler, J. R., Kulick, J. D. \& Eaton, J. K. 1994 Preferential concentration of heavy particles in a turbulent channel flow. Phys. Fluids 6, 3742-3749.

Gardiner, C. W. 2002 Handbook of Stochastic Methods. Springer.

Germano, M. 1986 Differential filters for the large eddy simulation of turbulent flows. Phys. Fluids 29, $1755-1757$.

Germano, M. 1992 Turbulence: the filtering approach. J. Fluid Mech. 238, 325-336.

Gore, R. A. \& Crowe, C. T. 1989 Effect of particle size on modulating turbulent intensity. Intl J. Multiphase Flow 15, 279-285.

Ijzermans, R. H. A., Hagmeijer, R. \& van Langen, P. J. 2007 Accumulation of heavy particles around a helical vortex filament. Phys. Fluids 19, 107102.

Jones, W. P. \& Wille, M. $1996 a$ Large eddy simulation of a plane jet in a cross flow. Intl J. Heat Fluid Flow 17, 296-306.

JONES, W. P. \& WiLLE, M. $1996 b$ Large eddy simulation of a round jet in a cross-flow. In Engineering Turbulence Modelling and Experiments 3 (ed. W. Rodi \& G. Bergeles), pp. 199-208. Heraklion, Crete.

KAISER, G. 1994 A Friendly Guide to Wavelets. Birkhäuser.

KidA, S. \& OHKITANi, K. 1992 Spatiotemporal intermittency and instability of a forced turbulence. Phys. Fluids 4, 1018-1027.

KLEIN, M., SADIKI, A. \& JANICKA, J. 2003 A digital filter based generation of inflow data for spatially developing direct numerical or large eddy simulations. J. Comput. Phys. 186, 652-665.

Klimontovich, I. L. 1969 The Statistical Theory of Non-equilibrium Processes in a Plasma. Pergamon.

Kloeden, P. E. \& Platen, E. 1992 Numerical Solution of Stochastic Differential Equations. Springer.

Kobayashi, H., Masutani, S. M., Azuhata, S., Arashi, N. \& Hishinuma, Y. 1988 Transport Phenomena in Turbulent Flows - Theory, Experiment and Simulations (ed. M. Hirata \& N. Kasagi) pp. 433-446. Hemisphere.

Kulick, J. D., Fessler, J. R. \& Eaton, J. K. 1994 Particle response and turbulence modification in fully developed channel flow. J. Fluid Mech. 277, 109-134.

La Porta, A., Voth, G. A., Crawford, A. M., Alexander, J. \& Bodenschatz, E. 2002 Fluid particle accelerations in fully developed turbulence. Nature 409, 1017-1019.

Lazaro, B. J. \& Lasheras, J. C. 1989 Particle dispersion in a turbulent, plane, free shear layer. Phys. Fluids A 1, 1035-1044.

Lazaro, B. J. \& Lasheras, J. C. $1992 a$ Particle dispersion in the developing free shear layer. Part 1. Unforced flow. J. Fluid Mech. 235, 135-178.

Lazaro, B. J. \& Lasheras, J. C. $1992 b$ Particle dispersion in the developing free shear layer. Part 2. Forced flow. J. Fluid Mech. 235, 179-221.

Lefebvre, A. H. 1989 Atomization and Sprays. Hemisphere.

Liboff, R. L. 1998 Kinetic Theory: Classical, Quantum and Relativistic Descriptions. Prentice-Hall.

Longmire, E. K. \& Eaton, J. K. 1992 Structure of a particle-laden round jet. J. Fluid Mech. 236, $217-257$.

Lund, T. S., Wu, X. \& SQuires, D. 1998 Generation of turbulent inflow data for spatially-developing boundary layer simulations. J. Comput. Phys. 140, 233-258.

Lundgren, T. 1967 Distribution functions in the statistical theory of turbulence. Phys. Fluids 10 (5), 969-975.

Marcu, B. \& Meiburg, E. $1996 a$ The effect of streamwise braid vortices on the particle dispersion in a plane mixing layer. I. Equilibrium points and their stability. Phys. Fluids 8, 715-733.

Marcu, B. \& Meiburg, E. $1996 b$ Three-dimensional features of particle dispersion in a nominally plane mixing layer. Phys. Fluids 8, 2266-2268.

Marcu, B., Meiburg, E. \& Raju, N. 1996 The effect of streamwise braid vortices on the particle dispersion in a plane mixing layer. II. Nonlinear particle dynamics. Phys. Fluids 8, 734-753.

DI MARE, F. 2002 Large eddy simulation of reacting and non-reacting flows in complex geometries. $\mathrm{PhD}$ thesis, Imperial College, University of London, London, UK.

Di Mare, L. \& Jones, W. P. 2003 LES of turbulent flow past a swept fence. Intl J. Heat Fluid Flow 24, 606-615. 
Di Mare, L., Klein, M., Jones, W. P. \& Janicka, J. 2006 Synthetic turbulence inflow conditions for large eddy simulation. Phys. Fluids 18, 025107.

Martin, E. \& Meiburg, E. 1994 The accumulation and dispersion of heavy particles in forced two-dimensional mixing layers. i. The fundamental and sub harmonic cases. Phys. Fluids 6, $1116-1132$.

MashayeK, F. \& Pandya, R. V. R. 2003 Analytical description of particle/droplet laden turbulent flows. Prog. Energy Combust. Sci. 29, 329-378.

MAXeY, M. \& Riley, J. J. 1983 Equation of motion for a small rigid sphere in a nonuniform flow. Phys. Fluids 26, 883-889.

MAXEY, M. R. 1987 The gravitational settling of aerosol particles in homogeneous turbulence and random flow fields. J. Fluid Mech. 174, 441-465.

Miller, R. S. \& Bellan, J. 2000 Direct numerical simulation and sub-grid of transitional droplet laden temporal mixing layer. Phys. Fluids 12, 650-671.

Minier, J. \& Peirano, E. 2001 The pdf approach to turbulent polydispersed two phase flows. Phys. Rep. 352, 1-214.

Mordant, N., Metz, P., Michel, O. \& Pinton, J. 2001 Measurement of Lagrangian velocity in fully developed turbulence. Phys. Rev. Lett. 87, 214501.

OKong'o, N. \& BeLlan, J. 2000 A priori sub-grid analysis of temporal mixing layers with evaporating droplets. Phys. Fluids 12, 1573-1591.

Okong'o, N. \& Bellan, J. 2004 Consistent large-eddy simulation of a temporal mixing layer with evaporating drops. Part 1: Direct numerical simulation, formulation and a priori analysis. J. Fluid Mech. 499, 1-47.

Piomelli, U. 1999 Large eddy simulation: achievements and challenges. Prog. Aerospace Sci. 35, 335-362.

Piomelli, U. \& LiU, J. 1995 Large eddy simulation of rotating channel flows using a localised dynamic model. Phys. Fluids 7(4), 893-848.

Raju, N. \& Meiburg, E. 1995 The accumulation and dispersion of heavy particles in forced two-dimensional mixing layers. Part 2: The effect of gravity. Phys. Fluids 7, 1241-1264.

ReEKs, M. W. 1980 Eulerian direct interaction applied to the statistical motion of particles in a turbulent fluid. J. Fluid Mech. 97, 569-590.

Reeks, M. R. 1991 On a kinetic equation for the transport of particles in turbulent flows. Phys. Fluids A 3, 446-456.

ReEks, M. R. 1992 On the continuum equations for dispersed particles in nonuniform flows. Phys. Fluids A 4, 1291-1303.

Reeks, M. R. 1993 On the constitutive relations for dispersed particles in nonuniform flows. I: Dispersion in a simple shear flow. Phys. Fluids A 5, 750-761.

Righetti, M. \& Romano, G. P. 2004 Particle-fluid interactions in a plane near-wall turbulent flow. J. Fluid Mech. 505, 93-121.

Risken, H. 1984 The Fokker Planck Equation. Springer.

Scheffer, R. W., Hartmann, V. \& Dibble, R. W. 1987 Conditional sampling of velocity in a turbulent non premixed propane jet. AIAA J. 25, 1318-1330.

Schmidt, H. \& Schumann, U. 1989 Coherent structure of the convective boundary layer derived from large-eddy simulation. J. Fluid Mech. 200, 511-562.

Sirignano, W. A. 1999 Fluid Dynamics and Transport of Droplet and Sprays. Cambridge University Press.

Smagorinsky, J. 1963 General circulation experiments with the primitive equations. I- the basic experiment. Mon. Weather Rev. 91, 99-165.

Squires, K. D. \& Eaton, J. K. 1990 Particle response and turbulence modification in isotropic turbulence. Phys. Fluids A 2, 1191-1203.

Squires, K. D. \& Eaton, J. K. 1991 Preferential concentration of particles by turbulence. Phys. Fluids A 3, 1169-1178.

Steinstrasser, D. 1994 Large eddy simulation of fully developed pipe flow. Master's thesis, Diplomarbeit, Imperial College, London.

Subramaniam, S. 2000 Statistical representation of a spray as a point process. Phys. Fluids 12, 2413-2431.

Subramaniam, S. 2001a Statistical modeling of sprays using the droplet distribution function. Phys. Fluids 13, 624-642. 
Subramaniam, S. $2001 b$ Erratum: Statistical modeling of sprays using the droplet distribution function. Phys. Fluids 13, 2743.

Thomson, D. J. 1987 Criteria for the selection of stochastic models of particles trajectories in turbulent flows. J. Fluid Mech. 180, 529-556.

Torrence, C. \& Compo, G. P. 1998 A practical guide to wavelet analysis. Bull. Am. Met. Soc. 79, $61-78$.

Voth, G. A., La Porta, A., Crawford, A. M., Alexander, J. \& Bodenschatz, E. 2002 Measurement of particle accelerations in fully developed turbulence. J. Fluid Mech. 469, 121-160.

WANG, P. \& MAXEY, M. R. 1993 Settling velocity and concentration distribution of heavy particles in homogeneous isotropic turbulence. J. Fluid Mech. 256, 27-68.

Wen, F., Kamalu, N., Chung, J. N., Crowe, C. T. \& Troutt, T. R. 1992 Particle dispersion by vortex structures in plane mixing layers. Trans. ASME I: J. Fluids Engng 4, 657.

Wille, M. 1997 Large eddy simulation of jets in cross flows. PhD thesis, Department of Chemical Engineering, Imperial College of Science, Technology and Medicine, London SW7 2BY.

Williams, F. A. 1958 Spray combustion and atomization. Phys. Fluids 1, 541-545.

Williams, F. A. 1985 Combustion Theory: The Theory of Chemically Reacting Flow Systems. Benjamin Cummings.

Yaglom, A. M. 1957 Some classes of random fields in $n$-dimensional space. Theory Prob. Applics. 3, 273-320.

Yanenko, N. N. 1971 The Method of Fractional Steps. Springer.

Yuu, S., Ueno, T. \& UmeKage, T. 2001 Numerical simulation of the high Re number slit nozzle gas-particle jet using sub-grid scale coupling LES. Chem. Engng Sci 56, 4293-4307.

Zeff, B., Lanterman, D., McAllister, R., Roy, R., Kostelich, E. \& Lathrop, D. 2003 Measuring intense rotation and dissipation in turbulent flows. Nature 421, 146-149. 